\title{
Patients Generally May Return to Driving 4 Weeks After Hip Arthroscopy and 6 Weeks After Knee Arthroscopy: A Systematic Review and Meta-analysis
}

\author{
Samantha Palma, Vasileios Giannoudis, MRes, MBChB, Purva Patel, Jeya Palan, Ph.D., \\ Stephen Guy, MBChB, Hemant Pandit, D Phil, and Bernard Van Duren, D Phil
}

\begin{abstract}
Purpose: To consolidate the evidence from the available literature and undertake a meta-analysis to provide a reference for physicians to make evidence-based recommendations to their patients regarding the return to driving after hip or knee arthroscopic procedures. Methods: A systematic review was conducted using Preferred Reporting Items for Systematic Reviews and Meta-analyses (PRISMA) guidelines. The OVID, Embase, and Cochrane databases were searched through June 2020 for articles containing keywords and/or MeSH (Medical Subject Headings) terms "hip arthroscopy" and "knee arthroscopy" in conjunction with "total brake response time" or "reaction time" in the context of automobile driving. A title review and full article review were performed to assess quality and select relevant articles. A meta-analysis of qualifying articles was undertaken. Results: Eight studies met the inclusion criteria for meta-analysis of brake reaction time (BRT). Meta-analysis of all knee BRTs showed times slower than or equal to baseline BRTs through 5 weeks, with a trend of improving BRTs from 6 to 10 weeks (weeks 8 and 10 were significant, $P<.05$ ). Among all hip BRTs, week 2 showed times slower than baseline BRTs, but after week 4, a trend toward faster BRTs was observed through week 8 (week 8 was significant, $P<.05$ ). Conclusions: BRTs met baseline or control values and continued to improve after 6 weeks after knee arthroscopy and after 4 weeks after hip arthroscopy. On the basis of these results, it would be safe to recommend a return to driving at 6 weeks after knee arthroscopic procedures and 4 weeks after hip arthroscopic procedures. Clinical Relevance: These results can be used by surgeons to base their recommendations on to provide guidance for their patients on the resumption of driving. Although BRT is an important aspect of driving ability, there are additional factors that need to be taken into consideration when making these recommendations, including cessation of opioid analgesics, strength of the surgical limb, and range of motion.
\end{abstract}

$\mathbf{H}$ ip and knee arthroscopies are some of the most common orthopaedic procedures performed. Studies in the United States have suggested that 70,000 hip arthroscopies are performed annually, with a further 984,607 knees arthroscopies. ${ }^{1,2}$ Such procedures have many advantages over their open-procedure

From Indiana University School of Medicine, Indianapolis, Indiana, U.S.A. (S.P., P.P.); Leeds Orthopaedic $\theta$ Trauma Sciences, School of Medicine, University of Leeds, Leeds, England (V.G.); Leeds Institute of Rheumatic and Musculoskeletal Medicine, University of Leeds, Leeds, England (J.P., H.P., B.v.D.); and Bradford Royal Infirmary, Bradford, England (S.G.).

The authors report the following potential conflicts of interest or sources of funding: This project was funded, in part, with support from Indiana University School of Medicine-West Lafayette. The content is solely the responsibility of the authors and does not necessarily represent the official views of Indiana University School of Medicine-West Lafayette. The views expressed in this article are those of the authors and not necessarily those of the Biomedical Research Centre (BRC), National Institute for Health Research (NIHR), or Department of Health and Social Care. H.P. is an NIHR Senior Investigator. In addition, H.P. is a consultant for Zimmer Biomet, JRI, Medacta International, DePuy Synthes, Smith o Nephew, Meril Life, and counterparts, such as less postoperative swelling, reduced pain, faster recovery time, and reduced risk of complications. ${ }^{3}$ Arthroscopy is available for the treatment of a wide variety of orthopaedic conditions, including anterior cruciate ligament (ACL) reconstruction, meniscectomy, labral repair, femoroacetabular

Invibio; provides expert testimony on Kennedy's Law; and receives travel/ accommodations/meeting expenses from Zimmer Biomet and Medacta International, outside the submitted work. B.v.D. is a BRC-NIHR Academic Clinical Lecturer, outside the submitted work. Full ICMJE author disclosure forms are available for this article online, as supplementary material.

Received December 9, 2020; accepted August 19, 2021.

Address correspondence to Vasileios Giannoudis, MRes, MBChB, Academic Department Trauma o Orthopaedic Surgery, School of Medicine, University of Leeds, Leeds, England.E-mail:vviannoudis@aol.com

(C) 2021 THE AUTHORS. Published by Elsevier Inc. on behalf of the Arthroscopy Association of North America. This is an open access article under the CC BY-NC-ND license (http://creativecommons.org/licenses/by-nc-nd/4.0/).

2666-061X/201874

https://doi.org/10.1016/j.asmr.2021.08.015 
impingement treatment, and removal of loose bodies, among many others. ${ }^{3,4}$ With patients eager to return to their normal functional level for work, for school, or otherwise, physicians often encounter the question "When can I drive?" at some point in the course of treating of their patients.

Driving involves complex and rapid skill sequences requiring the ability to interact simultaneously with both the vehicle and the external environment. Historically, driving impairment has been described as an increased time needed to perform an emergency stop ${ }^{5}$ (often tested as brake reaction time [BRT]); simulations to determine the BRT of a patient after surgery is neither a cost-effective nor practical solution to implement in every clinic or facility that offers arthroscopic surgery. One study found that most physicians recommended that their patients could return to driving after narcotics were discontinued and whenever the patients' postoperative symptoms would allow for driving. ${ }^{6}$ A study on self-regulation of driving found a significant correlation with self-regulation related to driving and the subject's perception of his or her overall health. ${ }^{7}$ However, it was also found that the study participants rated their overall health much higher than expected, suggesting that the patient's perception of health may not be the most reliable indicator of the ability to return to driving, adding yet another layer of complexity regarding when and how to make recommendations to return to driving for patients after arthroscopic surgery.

The effects of other orthopaedic surgical procedures, such as hip or knee arthroplasty, on the optimal return-todriving time have also been examined. Whereas the effects of these procedures on driving ability have been better documented, ${ }^{8}$ there is a paucity in the literature investigating the effects of hip and knee arthroscopy on driving ability. Among the studies that exist, the recommendations for the optimal time to return to driving are varied.

When making postoperative recommendations for the return to driving, the safety of the patient is paramount. Additionally, there are legal ramifications, as well as the potential of harm to other individuals, that are equally as important to consider when making these recommendations. Previous reviews have combined and presented the results and recommendations from existing studies, but none have completed a metaanalysis of the existing data to search for overarching trends or patterns. ${ }^{5,8-12}$

The purpose of this study was to consolidate the evidence from the available literature and undertake a meta-analysis to provide a reference for physicians to make evidence-based recommendations to their patients regarding the return to driving after hip or knee arthroscopic procedures. We hypothesized that driving ability would be adversely affected in the postoperative period after arthroscopic procedures, making a return to driving during this period unsafe for the patient.

\section{Methods}

\section{Search Strategy and Selection}

The procedures for this review were developed based on Preferred Reporting Items for Systematic Reviews and Meta-analyses (PRISMA) guidelines. ${ }^{13}$ The OVID, Embase, and Cochrane databases were all used for this systematic review. A brief review of related articles helped to determine the optimal keywords and MeSH (Medical Subject Headings) terms to be used. These terms were incorporated into the search protocol prior to data collection. The search protocol is shown in Appendix Table 1.

\section{Study Selection}

The inclusion criteria were established following the PICO (population-intervention-comparison-outcomes) approach: The population was defined as adults (age $\geq$ 18 years) undergoing hip or knee arthroscopy who were able to drive (in possession of a driver's license). The intervention was defined as hip or knee arthroscopy. The comparator was defined as the preoperative baseline or control cohort. Regarding outcomes, the primary outcome measured was the total brake response time. The secondary outcomes were reaction time, movement time, and braking force.

All studies published prior to July 1, 2020, were considered for eligibility. Table 1 summarizes the inclusion and exclusion criteria used. Titles and abstracts were screened for relevance prior to full inspection. The references of all included studies were reviewed to locate any articles that may have been missed in the database search. Duplicate articles between the databases were removed, and the full texts of all studies meeting the inclusion criteria were obtained.

\section{Statistical Analysis and Assessment of Methodologic Quality}

Data were extracted from each relevant study and analyzed using RevMan 5.4 (Review Manager, version 5.4; Cochrane, London, England). Means and standard deviations for BRTs were obtained from available study data for meta-analysis. BRTs were compared with preoperative or control values in 3 separate analyses; right (hip or knee) BRTs, left (hip or knee) BRTs, and all (hip or knee) BRTs. Standardized mean differences between preoperative or control BRTs and postoperative BRTs were assessed. When confidence intervals, standard errors, and $P$ values were given in place of standard deviations, ${ }^{14}$ standard deviations were imputed using the methods described in the Cochrane Handbook for Systematic Reviews of Interventions (chapter 7.7.3.3). ${ }^{15}$

With varying methodologies and outcome measures, heterogeneity among studies was considered likely, and a random-effects model was used for analysis. In all analyses, $P<.05$ was considered statistically significant. 
Table 1. Details of Inclusion and Exclusion Criteria for Selection of Included Articles

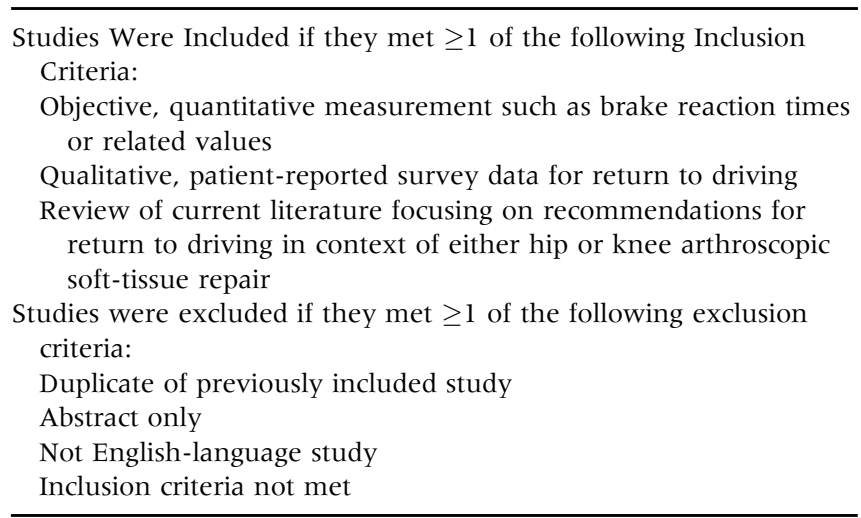

Additionally, the $I^{2}$ value was calculated to measure the heterogeneity of the included studies, indicating the percentage of variation in the meta-analysis caused by heterogeneity rather than chance. An $I^{2}$ value lower than $30 \%$ was considered low heterogeneity, whereas an $I^{2}$ value greater than $75 \%$ was considered high heterogeneity. ${ }^{15}$

Articles selected for the meta-analysis were critically appraised and examined for bias using the Critical Appraisal Skills Programme (CASP) checklist. ${ }^{16}$ Each study was graded as having a low, medium, or high risk of bias.

The methodologic quality of the included studies was assessed using the Methodological Index for Nonrandomized Studies (MINORS) index. ${ }^{17}$ The MINORS index produces a score between 0 and 24, with scores of 0 (not reported), 1 (reported but inadequate), or 2 (reported and adequate) being given for 12 separate factors. ${ }^{17}$ The score is designed to assess the methodologic quality of nonrandomized surgical studies, whether comparative or noncomparative, with the ideal score being 16 for noncomparative studies and 24 for comparative studies. ${ }^{17}$ The level of evidence was graded using the Oxford Centre for Evidence-Based Medicine (OCEBM) scale for levels of evidence. ${ }^{18}$

The overall quality of the evidence in the metaanalysis was assessed using the Grading of Recommendations, Assessment, Development and Evaluation (GRADE) system. ${ }^{19}$ Recommendations were classified as either high (we are very confident that the effect in the study reflects the actual effect), moderate (we are quite confident that the effect in the study is close to the true effect, but it is also possible that it is substantially different), low (the true effect may differ significantly from the estimate), or very low (true effect is probably markedly different from the estimated effect).

\section{Results}

The PRISMA flowchart is shown in Fig 1. After searches of the 3 databases and removal of duplicates, a total of 22 articles were identified. On a full article review, 4 articles were removed because of lack of relevance, abstract-only status, inability to access, and repeat of a previous study. One additional article was found based on a review of references from the previously collected articles. On the basis of the inclusion and exclusion criteria, a total of 19 relevant articles remained. The articles were classified as either prospective cohort, comparative, or case-control studies $^{14,20-26}$; repeated-measures design ${ }^{27}$; surveys or questionnaires $^{6,28}$; literature reviews ${ }^{8-10,28,29}$; or systematic reviews. ${ }^{11}$ Of the remaining studies, 8 contained quantitative data on BRTs and were included in the metaanalysis. Table 2 details the characteristics of all included studies.

\section{Methodologic Quality}

Table 3 provides an overview of the Critical Appraisal Skills Programme (CASP), MINORS, and Oxford Centre for Evidence-Based Medicine (OCEBM) bias assessments, which were completed for all of the studies included in the meta-analysis. Fourteen studies were graded as low risk; 2 studies, low/moderate risk; 2 studies, moderate risk; and 1 study, moderate/high risk.

Grading of Recommendations, Assessment, Development and Evaluation (GRADE) analysis (Appendix Table 2) showed the quality of evidence for most of the pooled analyses to be low or very low. On the basis of this, there is a possibility that the true effect may differ significantly from the estimate.

\section{Systematic Review}

There were 1,103 patients, with a total of 1,095 remaining after we accounted for loss to follow-up and participant dropout. In the studies that reported the mean age, there was a range of $22 \pm 4.61$ years $^{26}$ to 44 \pm 11.4 years. $^{22}$

Within the existing literature, there was a range of recommendations for optimal return-to-driving time after hip and knee arthroscopic procedures (Fig 2). The most frequent recommendation for the timing of the return to driving was 6 weeks postoperatively, as reported by Cooper ${ }^{29}$ and Fleury et al. ${ }^{10}$ after ACL reconstruction (either side) and by Ho and Furlan ${ }^{9}$ after right-sided ACL reconstruction specifically. This is consistent with recommendations made by Gotlin et al. ${ }^{27}$ and Nguyen et al. ${ }^{21}$ based on their prospective experimental studies. Wasserman et al. ${ }^{22}$ studied the BRTs of 3 different groups based on the type of surgical graft for ACL reconstruction. They, too, recommended waiting 6 weeks to return to driving after hamstring and bone-patellar tendon-bone autograft procedures but recommended waiting only 3 weeks after surgery for tibialis anterior autograft procedures. ${ }^{22}$ However, these suggestions differ from those of DiSilvestro et al., ${ }^{11}$ who recommended a return to driving after ACL reconstruction at 4 weeks postoperatively. Both Hau et al. ${ }^{20}$ 


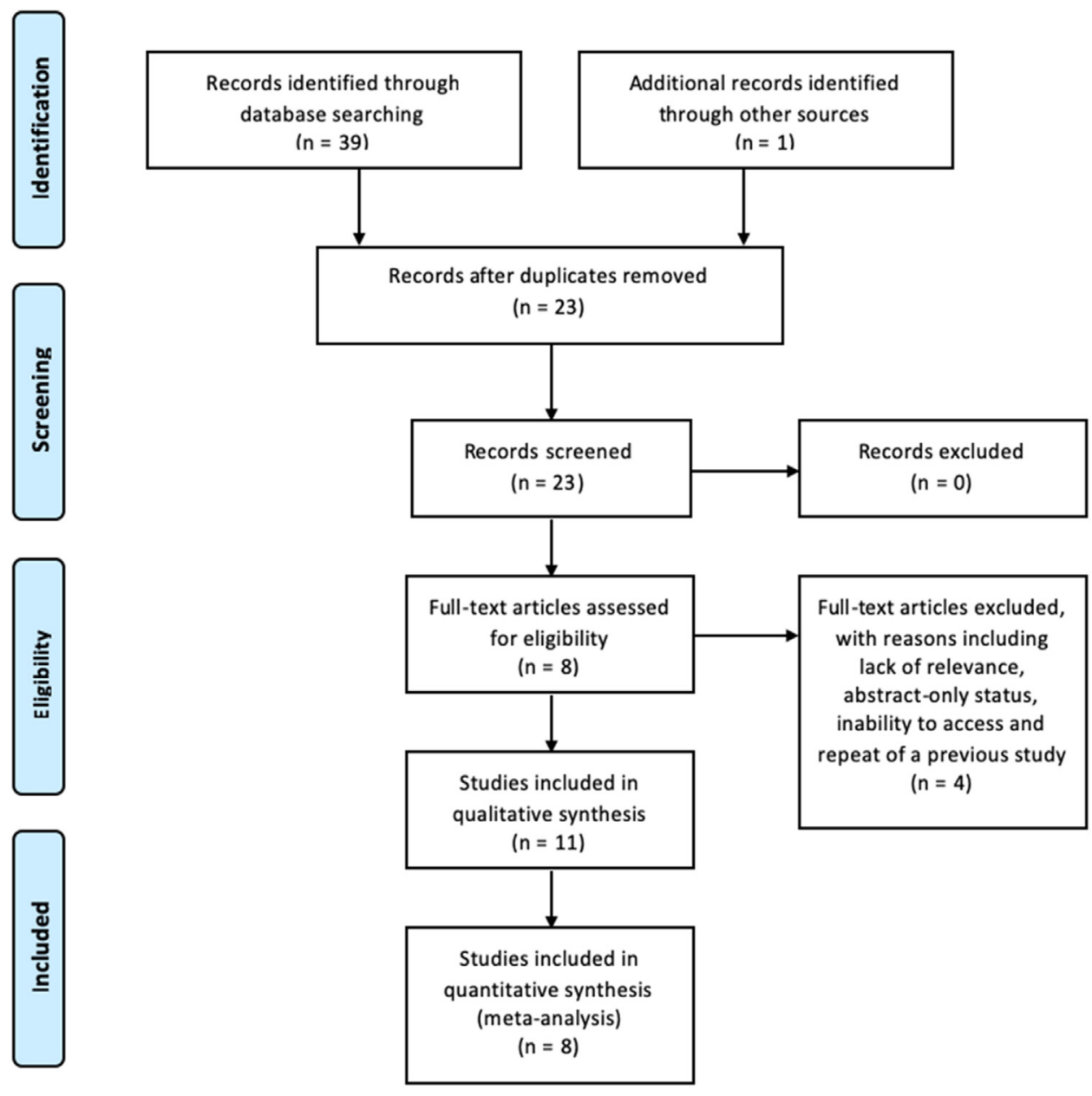

Fig 1. Preferred Reporting Items for Systematic Reviews and Meta-analyses (PRISMA) flow diagram for selection of articles used for systematic review and meta-analysis. and Argintar et al. ${ }^{6}$ suggested waiting at least 1 week to drive after simple knee arthroscopy (meniscectomy, chondroplasty, and diagnostic arthroscopy).

In a survey of surgeons performing right knee arthroscopy and their patients, Argintar et al. ${ }^{6}$ found that just under $30 \%$ of physicians had incorporated guidance on the return to driving during each of the preoperative patient visits. Of the physicians, 57\% initiated this conversation half of the time or less frequently and $33.4 \%$ initiated this conversation a quarter of the time or less frequently. ${ }^{6}$ Most of the physicians surveyed recommended to patients that they could return to driving once pain medications (i.e., opioid analgesics) ceased and when their postoperative symptoms allowed for it. Of the patients surveyed, only $8 \%$ stated that their doctor gave them advice regarding returning to driving. When they did receive advice, a large majority $(88 \%)$ followed the advice they were given. By use of a linear regression model and dummy variables, it was found that patients who were given 2 pieces of advice displayed a longer time to driving than did patients given a only 1 piece of advice. ${ }^{6}$ Of note, a study that examined early functional milestones after ACL reconstruction discovered predictors of an earlier return to driving, which included higher age, male sex, and left-sided surgery. ${ }^{30}$

Regarding hip arthroscopy, there was also a varied distribution of recommendations. Vera et al. ${ }^{23}$ recommended a return 2 weeks after left hip arthroscopy and 6 weeks after right hip arthroscopy. Momaya et al. ${ }^{25}$ similarly recommended a return to driving after 2 weeks but did not differentiate between left- and rightsided surgery. With a recommendation falling in the middle, Balazs et al. ${ }^{14}$ recommended returning to driving 4 weeks after hip arthroscopy, also not differentiating by side of surgery. In a survey of patients in 3 different groups based on surgical approach (simultaneous treatment, staged treatment, or single-hip treatment), Mei-Dan et al. ${ }^{31}$ found that there was no significant difference in the time after surgery when patients returned to driving. They also noted that the return to daily activities in the bilateral surgery group was similar to that in the unilateral surgery group, "with the advantage of a single rehabilitation." ${ }^{31}$ 
Table 2. Characteristics of All Included Studies in Systematic Review

\begin{tabular}{|c|c|c|c|c|c|c|c|}
\hline Study Title & Authors & Type of Study & Year Published & Participants, $\mathrm{n}$ & Mean Age, yr & Timeline & Outcomes \\
\hline $\begin{array}{l}\text { Return to Driving After Hip } \\
\text { Arthroscopy }\end{array}$ & Momaya et al. ${ }^{25}$ & $\begin{array}{l}\text { Prospective study } \\
\text { (cohort) }\end{array}$ & 2018 & $\begin{array}{l}14 \text { (4 participants } \\
\text { dropped out) }\end{array}$ & $\begin{array}{l}27.39 \pm 9.13 \text { in surgical } \\
\text { group and } 28.35 \pm \\
5.81 \text { in control group }\end{array}$ & $\begin{array}{l}\text { The study period was } \\
\text { October } 2014 \text { to } \\
\text { November } 2015 \text {, } \\
\text { with follow-up } \\
\text { performed every } 2 \\
\text { wk for a total of } 8 \mathrm{wk} \text {. }\end{array}$ & Right BRTs \\
\hline $\begin{array}{l}\text { Evaluation of Driving Skills } \\
\text { After Anterior Cruciate } \\
\text { Ligament Reconstruction } \\
\text { With Hamstring } \\
\text { Autograft }\end{array}$ & Valentí et al. ${ }^{24}$ & $\begin{array}{l}\text { Prospective study } \\
\text { (cohort) }\end{array}$ & 2018 & 62 & $\begin{array}{l}32.39 \pm 9.27 \text { in surgical } \\
\text { group and } 28.58 \pm \\
8.91 \text { in control group }\end{array}$ & $\begin{array}{l}\text { Subjects completed } \\
\text { simulated driving and } \\
\text { reaction time tests } \\
\text { between 4-6 wk } \\
\text { postoperatively. }\end{array}$ & Right and left BRTs \\
\hline $\begin{array}{l}\text { Measurement of Brake } \\
\text { Response Time After } \\
\text { Right Anterior Cruciate } \\
\text { Ligament Reconstruction }\end{array}$ & Gotlin et al. ${ }^{27}$ & $\begin{array}{l}\text { Prospective } \\
\text { repeated-measure } \\
\text { design }\end{array}$ & 2000 & 35 & $\begin{array}{l}31.2 \text { in surgical group } \\
\text { and } 29.3 \text { in control } \\
\text { group }\end{array}$ & $\begin{array}{l}\text { Multiple variables were } \\
\text { measured every } 2 \mathrm{wk} \\
\text { for a total of } 10 \mathrm{wk} \\
\text { postoperatively. }\end{array}$ & Right BRTs \\
\hline $\begin{array}{l}\text { Reaction Time and Brake } \\
\text { Pedal Depression } \\
\text { Following Arthroscopic } \\
\text { Hip Surgery: A } \\
\text { Prospective Case-Control } \\
\text { Study }\end{array}$ & Balazs et al. ${ }^{14}$ & $\begin{array}{l}\text { Prospective case- } \\
\text { control study }\end{array}$ & 2018 & 118 & $\begin{array}{l}33.7 \text { in surgical group } \\
\text { and } 33.3 \text { in control } \\
\text { group }\end{array}$ & $\begin{array}{l}\text { BRT and BPD were } \\
\text { measured } \\
\text { preoperatively and at } \\
2,4 \text {, and } 6 \mathrm{wk} \\
\text { postoperatively. }\end{array}$ & Right and left BRTs \\
\hline $\begin{array}{l}\text { Brake Reaction Time After } \\
\text { Hip Arthroscopy for } \\
\text { Femoroacetabular } \\
\text { Impingement and Labral } \\
\text { Tear }\end{array}$ & Vera et al. ${ }^{23}$ & $\begin{array}{l}\text { Prospective } \\
\text { comparative } \\
\text { study (Level II, } \\
\text { diagnostic) }\end{array}$ & 2017 & $\begin{array}{l}40 \text { ( } 2 \text { participants } \\
\text { failed to meet } \\
\text { testing time frame } \\
\text { and were excluded) }\end{array}$ & $\begin{array}{l}37.1 \pm 12.7 \text { for right } \\
\text { knee, } 32.1 \pm 9.2 \text { for } \\
\text { left knee, } 35.5 \pm 11.1 \\
\text { for right control, and } \\
32.6 \pm 8.4 \text { for left } \\
\text { control }\end{array}$ & $\begin{array}{l}\text { BRTs and sit-to-stand } \\
\text { numbers were } \\
\text { measured } \\
\text { preoperatively and } \\
\text { every } 2 \mathrm{wk} \\
\text { postoperatively for a } \\
\text { total of } 8 \mathrm{wk} \text {. }\end{array}$ & $\begin{array}{l}\text { Right and left BRTs } \\
\text { and sit-to-stand } \\
\text { scores }\end{array}$ \\
\hline $\begin{array}{l}\text { Braking Reaction Time } \\
\text { After Right-Knee } \\
\text { Anterior Cruciate } \\
\text { Ligament } \\
\text { Reconstruction: A } \\
\text { Comparison of } 3 \text { Grafts }\end{array}$ & Wasserman et al. ${ }^{22}$ & Case-control study & 2017 & 57 & $\begin{array}{l}29.2 \pm 8.2 \text { for HS, } 25.0 \\
\pm 4.2 \text { for BPTB, } 44.0 \\
\pm 11.4 \text { for TA, and } \\
30.4 \pm 3.8 \text { for control }\end{array}$ & $\begin{array}{l}\text { Thirty healthy } \\
\text { volunteers were } \\
\text { tested during } 1 \text { visit } \\
\text { to determine normal } \\
\text { mean values, and } 27 \\
\text { treatment subjects } \\
\text { were tested at } 1,3, \\
\text { and } 6 \text { wk after ACL } \\
\text { reconstruction. }\end{array}$ & Right BRTs \\
\hline $\begin{array}{l}\text { Driving Reaction Time } \\
\text { Before and After } \\
\text { Anterior Cruciate } \\
\text { Ligament Reconstruction }\end{array}$ & Nguyen et al. ${ }^{21}$ & $\begin{array}{l}\text { Cohort, prospective, } \\
\text { comparative } \\
\text { study }\end{array}$ & 2000 & 73 & $\begin{array}{l}30.2 \pm 7.9 \text { for right } \\
\text { knee, } 30.3 \pm 8.5 \text { for } \\
\text { left knee, and } 33.8 \pm \\
10.8 \text { in control group }\end{array}$ & $\begin{array}{l}\text { Each patient } \\
\text { underwent driving } \\
\text { reaction time and } \\
\text { stand tests both } \\
\text { preoperatively and at } \\
2,4,6, \text { and } 8 \mathrm{wk} \\
\text { postoperatively. }\end{array}$ & Right and left BRTs \\
\hline
\end{tabular}




\begin{tabular}{|c|c|c|c|c|c|c|c|}
\hline Study Title & Authors & Type of Study & Year Published & Participants, n & Mean Age, yr & Timeline & Outcomes \\
\hline $\begin{array}{l}\text { Driving Reaction Time } \\
\text { After Right Knee } \\
\text { Arthroscopy }\end{array}$ & Hau et al. ${ }^{20}$ & $\begin{array}{l}\text { Cohort, prospective, } \\
\text { comparative } \\
\text { study }\end{array}$ & 2000 & 55 & $\begin{array}{l}42.2 \pm 14.2 \text { in surgical } \\
\text { group and } 33.6 \pm \\
11.0 \text { control }\end{array}$ & $\begin{array}{l}\text { Each patient was tested } \\
\text { both preoperatively } \\
\text { and at } 1 \mathrm{wk} \text { and } 4 \mathrm{wk} \\
\text { after arthroscopy. }\end{array}$ & Right BRTs \\
\hline $\begin{array}{l}\text { A Comparative Study of } \\
\text { the Neuromuscular } \\
\text { Response During a } \\
\text { Dynamic Activity After } \\
\text { Anterior Cruciate } \\
\text { Ligament Reconstruction }\end{array}$ & Oliver et al. ${ }^{26}$ & $\begin{array}{l}\text { Prospective, } \\
\text { comparative, } \\
\text { matched, } \\
\text { controlled study }\end{array}$ & 2019 & $\begin{array}{l}25 \text { ( } 2 \text { participants } \\
\text { later dropped out) }\end{array}$ & $22 \pm 4.61$ & $\begin{array}{l}\text { Each patient } \\
\text { underwent } \\
\text { measurement } \\
\text { preoperatively and } \\
\text { again at } 4 \text { and } 6 \text { mo } \\
\text { postoperatively. }\end{array}$ & $\begin{array}{l}\text { Neuromuscular } \\
\text { response of } 5 \\
\text { different lower } \\
\text { limb muscles }\end{array}$ \\
\hline $\begin{array}{l}\text { Bilateral Hip Arthroscopy } \\
\text { Under the Same } \\
\text { Anesthetic for Patients } \\
\text { With Symptomatic } \\
\text { Bilateral } \\
\text { Femoroacetabular } \\
\text { Impingement: 1-Year } \\
\text { Outcomes }\end{array}$ & Mei-Dan et al. ${ }^{31}$ & $\begin{array}{l}\text { Retrospective } \\
\text { comparative } \\
\text { study }\end{array}$ & 2014 & 76 & 33 & $\begin{array}{l}\text { Patients were divided } \\
\text { into } 3 \text { groups based } \\
\text { on the approach to } \\
\text { hip arthroscopy } \\
\text { (simultaneous } \\
\text { treatment, staged } \\
\text { treatment, or single- } \\
\text { hip treatment). }\end{array}$ & $\begin{array}{l}\text { Time (day) of return } \\
\text { to various } \\
\text { activities and time } \\
\text { (day) of cessation } \\
\text { of medications }\end{array}$ \\
\hline $\begin{array}{l}\text { Examination of Early } \\
\text { Functional Recovery } \\
\text { After ACL } \\
\text { Reconstruction: } \\
\text { Functional Milestone } \\
\text { Achievement and Self- } \\
\text { Reported Function }\end{array}$ & Obermeier et al. ${ }^{30}$ & $\begin{array}{l}\text { Prospective, } \\
\text { longitudinal, } \\
\text { observational } \\
\text { study }\end{array}$ & 2018 & 182 & $28 \pm 12$ & $\begin{array}{l}\text { Data were collected via } \\
\text { survey before surgery } \\
\text { as well as } 1,2,4,8, \\
\text { and } 12 \text { wk after } \\
\text { surgery. }\end{array}$ & $\begin{array}{l}\text { SMFA scores, week } \\
\text { of return to } \\
\text { driving, and week } \\
\text { of cessation of } \\
\text { pain medication }\end{array}$ \\
\hline $\begin{array}{l}\text { Recommendations for } \\
\text { Driving After Right Knee } \\
\text { Arthroscopy }\end{array}$ & Argintar et al. ${ }^{6}$ & Survey & 2013 & $\begin{array}{l}266 \text { (197 doctors } \\
\text { and } 69 \text { patients) }\end{array}$ & - & $\begin{array}{l}\text { Questionnaires were } \\
\text { emailed to surgeons } \\
\text { and patients were } \\
\text { identified as having } \\
\text { undergone knee } \\
\text { arthroscopic surgical } \\
\text { procedures } \\
\text { (including any } \\
\text { combination of } \\
\text { partial } \\
\text { meniscectomy, } \\
\text { chondroplasty, or } \\
\text { debridement). Any } \\
\text { patients undergoing } \\
\text { additional } \\
\text { ligamentous } \\
\text { reconstruction, } \\
\text { microfracture, open } \\
\text { arthrotomy, }\end{array}$ & $\begin{array}{l}\text { Frequency of } \\
\text { recommendations } \\
\text { for postoperative } \\
\text { driving, when } \\
\text { physicians } \\
\text { recommend to } \\
\text { return to driving } \\
\text { postoperatively, } \\
\text { how often } \\
\text { patients look at } \\
\text { physicians' } \\
\text { advice, when } \\
\text { patients look at } \\
\text { physicians' } \\
\text { advice, and what } \\
\text { policies insurance } \\
\text { companies have } \\
\text { in place for return }\end{array}$ \\
\hline
\end{tabular}




Timeline

hardware

introduction, or any procedure on the left

knee were excluded

from the study.

Knee Arthroscopy and Lewis et al. ${ }^{28} \quad$ Review/prospective

Prospective

Questionnaire Survey

and Review of the

Literature

\section{Driving After Orthopaedic} Surgery

Resuming Motor Vehicle

Driving Following

Orthopaedic Surgery or

Limb Trauma

Clinical Decision Making:

Doctor, When Can I

Drive?

Driving Following Acute

Lower Limb Painful

Driving After Upper or

Lower Extremity

Orthopaedic Surgery

When Can I Drive After

Orthopaedic Surgery? A

Systematic Review

NOTE. A dash indicates data were not available in the study. Age is presented as mean \pm standard deviation

ACL, anterior cruciate ligament; BPD, brake pedal depression; BPTB, bone-patellar tendon-bone; BRT, brake reaction time; HS, hamstring; SMFA, Short Musculoskeletal Function Assessment; TA, tibialis anterior.
Marecek and Review 2013

Schafer $^{5}$ Review 2012

Cooper $^{29} \quad$ Review

Ho and Furlan ${ }^{9} \quad$ Review

MacKenzie et al. $^{8} \quad$ Review

DiSilvestro et al. ${ }^{11} \quad$ Systematic review

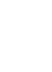

$-$

2007

2012

2019

2016
Patients were given

surveys at their 2-wk

Aspects of consent

follow-up

when patients

appointment.

driving, and

whether patients

experienced any

adverse events

$$
\text { - }
$$$$
\text { - }
$$$$
-
$$$$
\text { - }
$$$$
-
$$

$$
\text { - }
$$




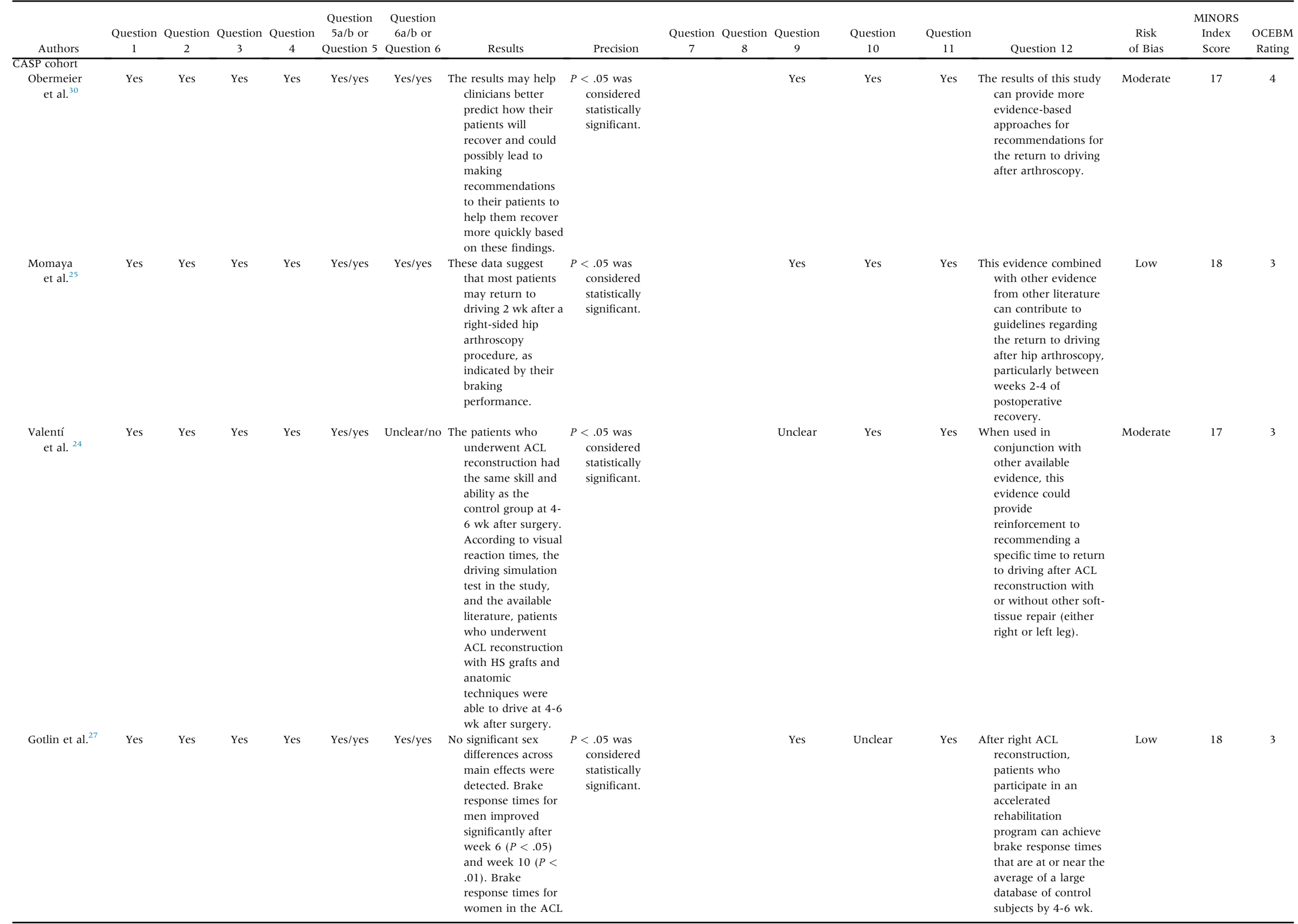


Table 3. Continued

\begin{tabular}{|c|c|c|c|c|c|c|c|c|c|c|c|c|c|c|c|c|c|}
\hline Authors & $\begin{array}{l}\text { Question } \\
\quad 1\end{array}$ & $\begin{array}{l}\text { Question } \\
2\end{array}$ & $\begin{array}{l}\text { Question } \\
3\end{array}$ & $\begin{array}{l}\text { Question } \\
\quad 4\end{array}$ & $\begin{array}{c}\text { Question } \\
5 \mathrm{a} / \mathrm{b} \text { or } \\
\text { Question } 5\end{array}$ & $\begin{array}{c}\text { Question } \\
\text { 6a/b or } \\
\text { Question } 6\end{array}$ & Results & Precision & $\begin{array}{l}\text { Question } \\
7\end{array}$ & $\begin{array}{l}\text { Question } \\
8\end{array}$ & $\begin{array}{l}\text { Question } \\
9\end{array}$ & $\begin{array}{c}\text { Question } \\
10\end{array}$ & $\begin{array}{l}\text { Question } \\
\quad 11\end{array}$ & Question 12 & $\begin{array}{c}\text { Risk } \\
\text { of Bias }\end{array}$ & $\begin{array}{l}\text { MINORS } \\
\text { Index } \\
\text { Score }\end{array}$ & $\begin{array}{c}\text { OCEBM } \\
\text { Rating }\end{array}$ \\
\hline & & & & & & & $\begin{array}{l}\text { treatment group } \\
\text { matched control } \\
\text { times at } 6 \text { wk. The } \\
6 \text {-m walk times for } \\
\text { control subjects } \\
\text { were faster than } \\
\text { those for the ACL } \\
\text { group } \\
\text { preoperatively ( } 2.6 \\
\text { seconds vs } 5.5 \\
\text { seconds) but } \\
\text { equalized by week } \\
\text { 6. It is important to } \\
\text { note that only the } \\
\text { male group met the } \\
\text { level of statistical } \\
\text { significance. }\end{array}$ & & & & & & & $\begin{array}{l}\text { The evidence (when } \\
\text { combined with other } \\
\text { evidence) may be } \\
\text { able to provide a } \\
\text { tentative guideline on } \\
\text { when patients can } \\
\text { safely return to } \\
\text { driving after right } \\
\text { ACL repair. } \\
\text { Significant sex-based } \\
\text { differences call for } \\
\text { further research. }\end{array}$ & & & \\
\hline 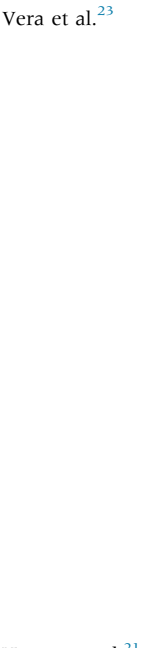 & Yes & Yes & Yes & Yes & Yes/yes & Yes/yes & $\begin{array}{l}\text { The study analyzed } 19 \\
\text { subjects (aged } 37.1 \\
\pm 12.7 \text { yr, } 10 \\
\text { women, } 11 \text { right } \\
\text { hips). All subjects } \\
\text { underwent } \\
\text { arthroscopic labral } \\
\text { repair and FAI } \\
\text { correction. There } \\
\text { was no difference } \\
\text { between the } \\
\text { preoperative BRT } \\
\text { (604 } \pm 148 \mathrm{ms)} \\
\text { and postoperative } \\
\text { BRTs (608 ms at } 2 \\
\text { wk, } 566 \mathrm{~ms} \text { at } 4 \\
\text { wk, } 559 \mathrm{~ms} \text { at } 6 \\
\text { wk, and } 595 \mathrm{~ms} \text { at } \\
8 \text { wk). There was } \\
\text { no difference } \\
\text { between controls } \\
\text { and subjects at any } \\
\text { time point. }\end{array}$ & $\begin{array}{r}P<.05 \text { was } \\
\text { considered } \\
\text { statistically } \\
\text { significant. } \\
\end{array}$ & & & Yes & Unclear & No & $\begin{array}{l}\text { The evidence could } \\
\text { possibly be used in } \\
\text { conjunction with } \\
\text { other evidence to } \\
\text { make } \\
\text { recommendations on } \\
\text { the return to driving } \\
\text { after hip arthroscopy; } \\
\text { however, a lack of } \\
\text { overall evidence } \\
\text { suggests the need for } \\
\text { more research on the } \\
\text { topic. }\end{array}$ & Moderate & 17 & 3 \\
\hline Nguyen et al. ${ }^{21}$ & Yes & Yes & Yes & Yes & Yes/yes & Yes/yes & $\begin{array}{l}\text { In the control group, } \\
\text { improvement was } \\
\text { seen in all } 3 \text { tests } \\
\text { over a period of } 8 \\
\text { wk, with marked } \\
\text { improvement after } \\
2 \text { wk. In the left } \\
\text { ACL group, the } \\
\text { stepping and } \\
\text { reaction time tests } \\
\text { showed a pattern of } \\
\text { improvement } \\
\text { similar to that in } \\
\text { the control group. } \\
\text { However, the } \\
\text { standing test }\end{array}$ & $\begin{array}{l}P<.05 \text { was } \\
\text { considered } \\
\text { statistically } \\
\text { significant. } \\
\\
\end{array}$ & & & Yes & Yes & Yes & $\begin{array}{l}\text { This study provides } \\
\text { quantitative evidence } \\
\text { on which to base } \\
\text { recommendations to } \\
\text { patients, although the } \\
\text { evidence is } 20 \mathrm{yr} \text { old. }\end{array}$ & Low & 18 & 3 \\
\hline
\end{tabular}




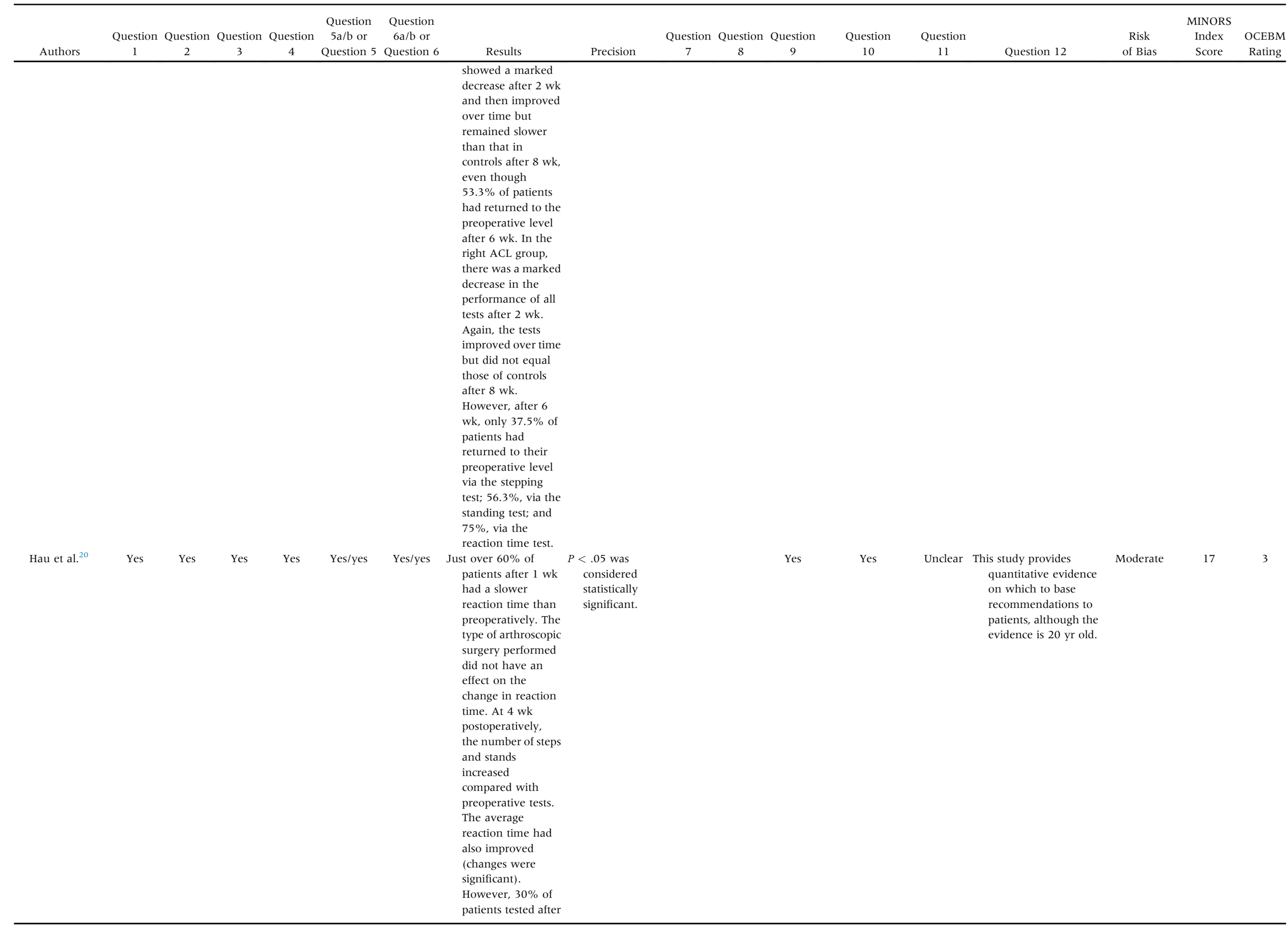


Table 3. Continued

\begin{tabular}{|c|c|c|c|c|c|c|c|c|c|c|c|c|c|c|c|c|c|}
\hline Authors & $\begin{array}{l}\text { Question } \\
\quad 1\end{array}$ & $\begin{array}{l}\text { Question } \\
\quad 2\end{array}$ & $\begin{array}{l}\text { Question } \\
\quad 3\end{array}$ & $\begin{array}{l}\text { Question } \\
\quad 4\end{array}$ & $\begin{array}{c}\text { Question } \\
5 \mathrm{a} / \mathrm{b} \text { or } \\
\text { Question } 5\end{array}$ & $\begin{array}{c}\text { Question } \\
\text { 6a/b or } \\
\text { Question } 6\end{array}$ & Results & Precision & $\begin{array}{l}\text { Question } \\
\quad 7\end{array}$ & $\begin{array}{l}\text { Question } \\
\quad 8\end{array}$ & $\begin{array}{l}\text { Question } \\
\quad 9\end{array}$ & $\begin{array}{l}\text { Question } \\
\quad 10\end{array}$ & $\begin{array}{l}\text { Question } \\
\quad 11\end{array}$ & Question 12 & $\begin{array}{c}\text { Risk } \\
\text { of Bias }\end{array}$ & $\begin{array}{l}\text { MINORS } \\
\text { Index } \\
\text { Score }\end{array}$ & $\begin{array}{c}\text { OCEBM } \\
\text { Rating }\end{array}$ \\
\hline $\begin{array}{l}\text { Mei-Dan } \\
\quad \text { et al. }{ }^{31}\end{array}$ & Yes & Yes & No & Unclear & Yes/yes & Yes/yes & $\begin{array}{l}4 \mathrm{wk} \text { still had } \\
\text { slower reaction } \\
\text { times than the } \\
\text { preoperative } \\
\text { reaction times. } \\
\text { The groups were not } \\
\text { randomized, some } \\
\text { crossover occurred, } \\
\text { and more patients } \\
\text { elected to undergo } \\
\text { simultaneous } \\
\text { approaches to hip } \\
\text { surgery, which } \\
\text { could lead to } \\
\text { favoring of the } \\
\text { results for that } \\
\text { category. }\end{array}$ & $\begin{array}{c}P<.05 \text { was } \\
\quad \text { considered } \\
\text { statistically } \\
\text { significant. }\end{array}$ & & & Unclear & Unclear & Unclear & $\begin{array}{l}\text { Simultaneous } \\
\text { femoroacetabular } \\
\text { impingement surgery } \\
\text { does not lead to } \\
\text { higher rates of } \\
\text { complications, } \\
\text { postoperative pain, } \\
\text { analgesic use, or side } \\
\text { effects. The return to } \\
\text { daily activities is } \\
\text { similar to that of a } \\
\text { single-hip procedure } \\
\text { with the advantage of } \\
\text { a single } \\
\text { rehabilitation. }\end{array}$ & Moderate & 16 & 3 \\
\hline $\begin{array}{l}\text { CASP case control } \\
\text { Oliver et al. }{ }^{26}\end{array}$ & Yes & Yes & Yes & Yes & Yes & Yes/yes & $\begin{array}{l}\text { Comparison of } \\
\text { reaction times in } \\
\text { the vastus medialis } \\
\text { showed that the } \\
\text { time for the injured } \\
\text { knee was longer } \\
\text { preoperatively but } \\
\text { it reduced over } \\
\text { time, reaching a } \\
\text { value at } 6 \text { mo } \\
\text { postoperatively } \\
\text { that was close to } \\
\text { the reaction time in } \\
\text { the uninjured knee } \\
\text { group. In the rectus } \\
\text { femoris, biceps } \\
\text { femoris, and } \\
\text { semitendinosus } \\
\text { muscles, the } \\
\text { reaction times in } \\
\text { the injured knee } \\
\text { group were similar } \\
\text { to those in the } \\
\text { uninjured knee } \\
\text { group at the } \\
\text { preoperative and } \\
\text { postoperative visits. }\end{array}$ & $\begin{array}{c}P<.05 \text { was } \\
\quad \text { considered } \\
\text { statistically } \\
\text { significant. }\end{array}$ & & & Yes & Unclear & Unclear & - & Moderate & 17 & 4 \\
\hline Balazs et al. ${ }^{14}$ & Yes & Yes & Yes & Yes & Yes & Yes/yes & $\begin{array}{l}\text { The results showed } \\
\text { that there was a } \\
\text { significantly } \\
\text { increased BRT in } \\
\text { patients } \\
\text { undergoing right } \\
\text { hip arthroscopy, } \\
\text { which reached }\end{array}$ & $\begin{array}{c}P<.05 \text { was } \\
\text { considered } \\
\text { statistically } \\
\text { significant. }\end{array}$ & & & Yes & Yes & Yes & - & Low & 18 & 3 \\
\hline
\end{tabular}




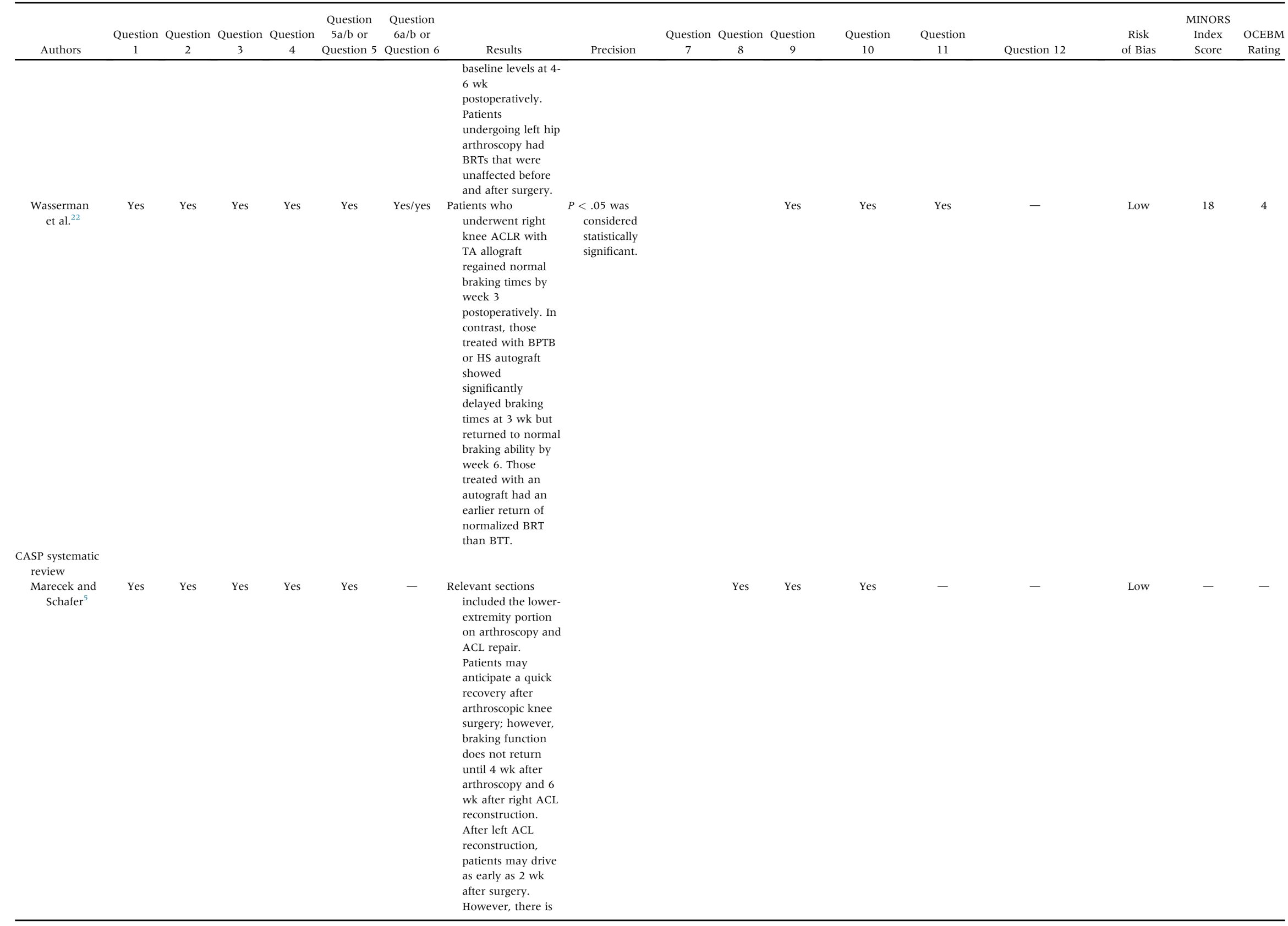




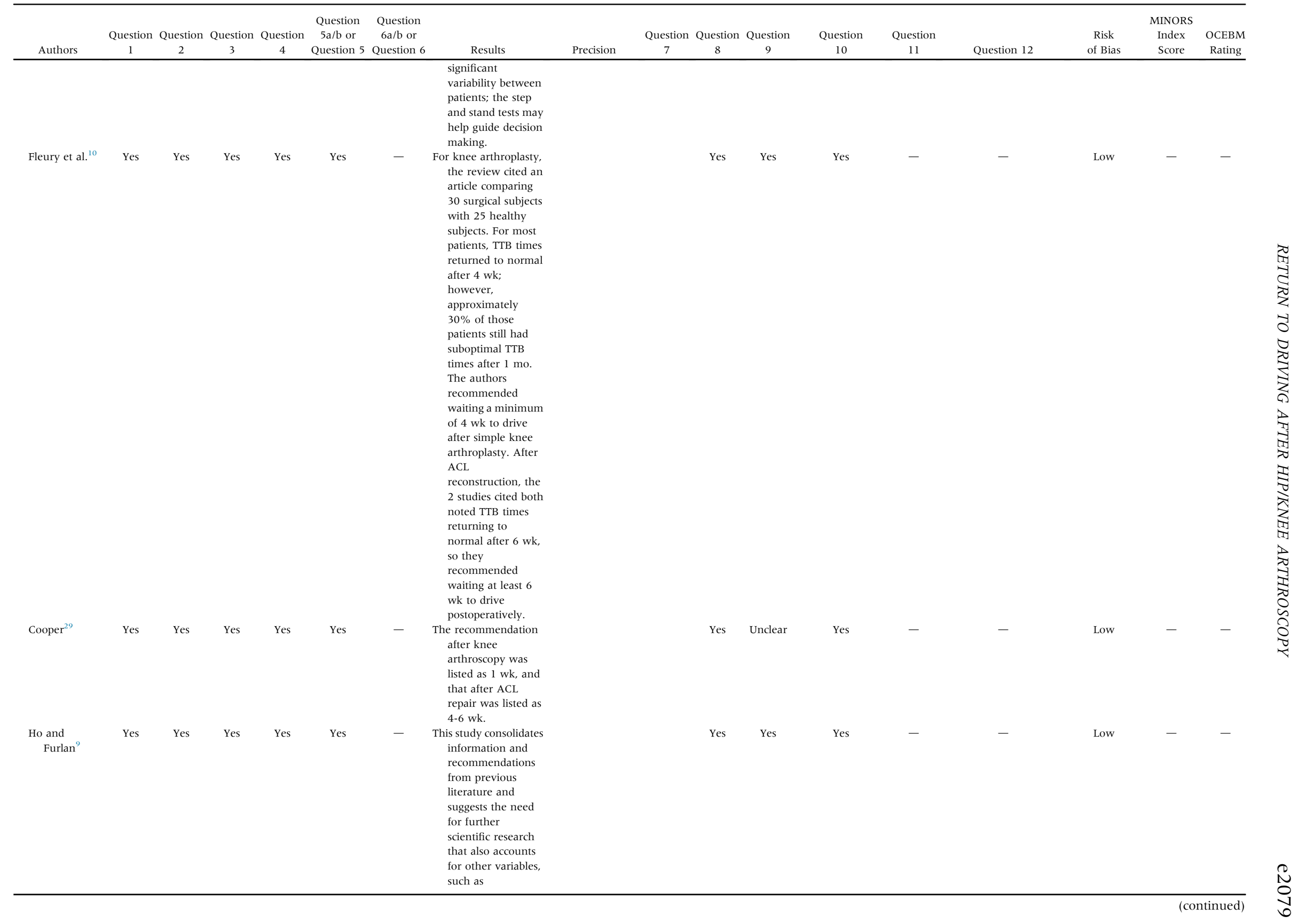




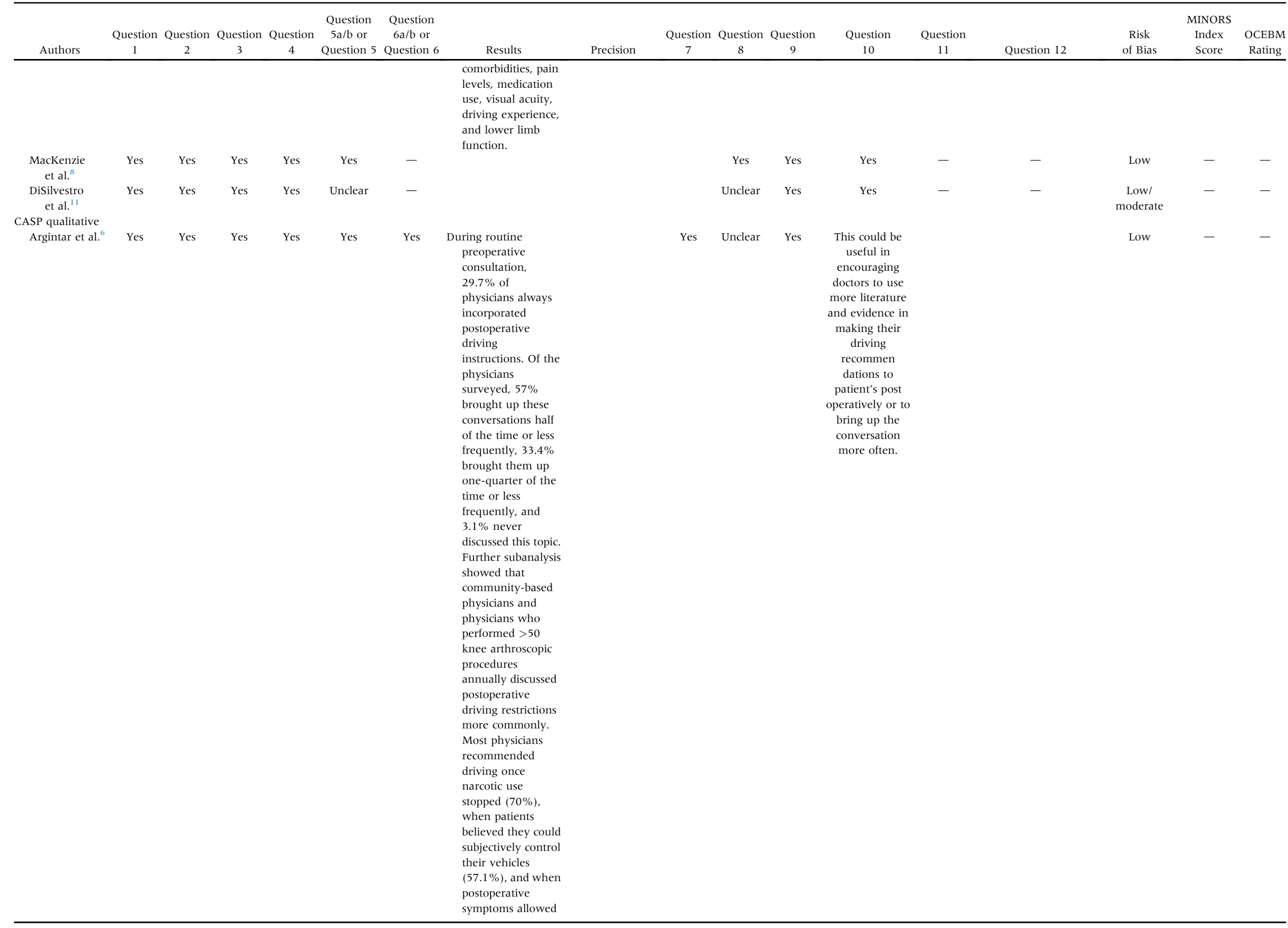




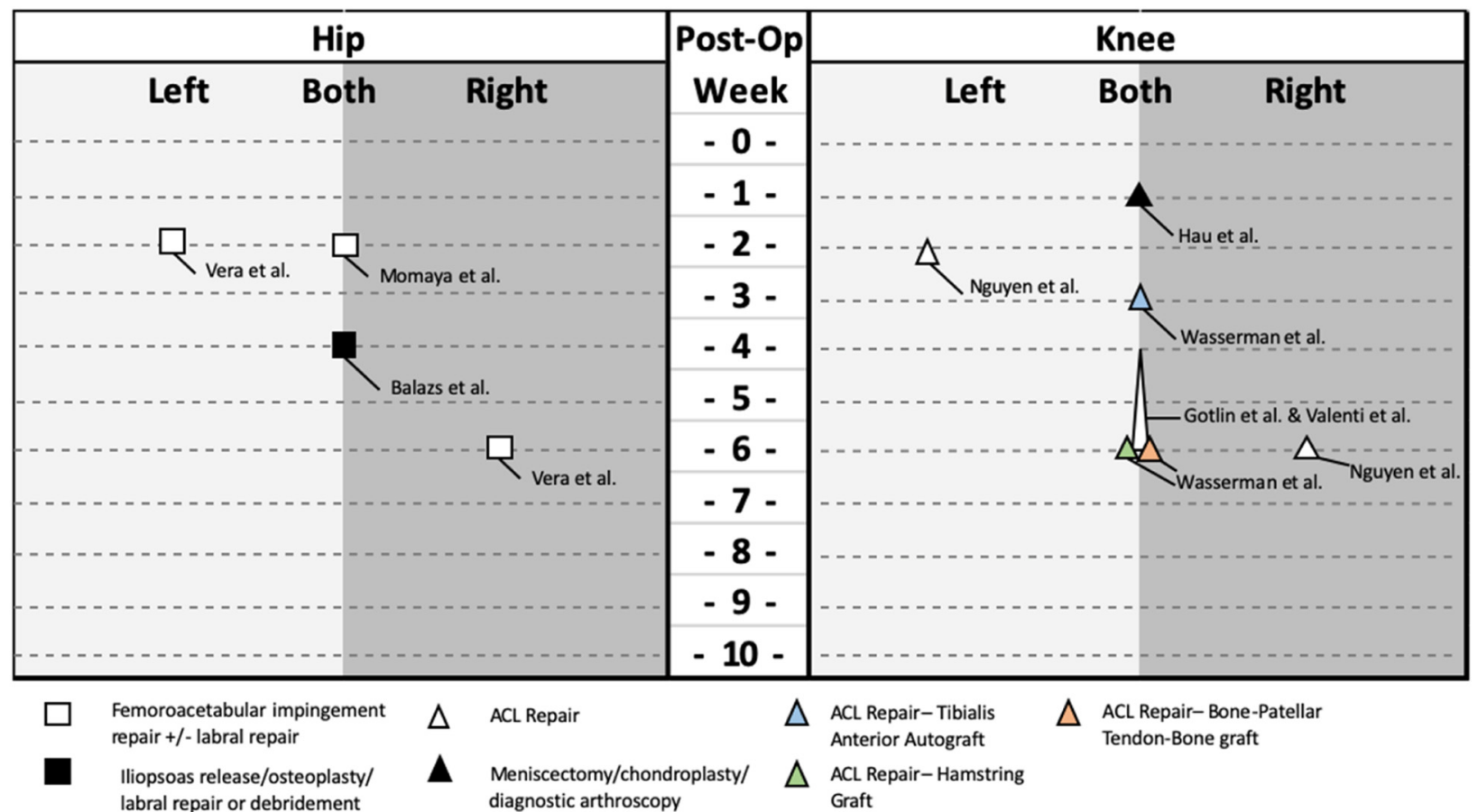

Fig 2. Summary of recommendations for return to driving per study: visual depiction of operative procedure performed and time required to return to driving. (ACL, anterior cruciate ligament; Post-Op, postoperative.)

\section{Meta-analysis}

The demographic characteristics of patients included in the meta-analysis are detailed in Table 4, and the results of each of the included studies are presented in Table 5. In total, 436 patients were included in the meta-analysis, with an age range from $28.6 \pm 8.91$ years $^{30}$ to $44 \pm 11.4$ years. $^{22}$ Of these studies, 5 were cohort studies, 2 were case-control studies, and 1 was a prospective comparative study. BRT data were extracted from all studies, and all data were pooled for analysis. Postoperative BRT data were compared with preoperative BRTs where available and with control group data when preoperative data were not provided. ${ }^{22,24,27}$

\section{Knee Arthroscopy}

For right knee arthroscopic procedures, collective means for week 1 after surgery showed average BRTs considerably slower than preoperative or control values. Weeks 2 through 10 showed a general trend of decreasing (faster) BRTs, although only weeks 5 and 10 showed statistically significant findings (Fig 3). In the left knee arthroscopy group, weeks 2 through 4 showed BRTs slower than or near baseline values, whereas weeks 6 through 8 showed improved average BRTs compared with preoperative or control values (Fig 4). None of the values were statistically significant. Within the group of combined left and right knee arthroscopies ("all knee BRTs"), BRTs were slower than or near baseline BRTs from week 1 through week 5 postoperatively. There was a trend of improving BRTs from week 6 through week 10, although only weeks 8 and 10 showed statistically significant findings (Fig 5).

\section{Hip Arthroscopy}

Within the right hip arthroscopy group, there was a clear trend of improving BRTs, with average BRTs being faster than baseline after week 4 (Fig 6). Left hip arthroscopy mean BRTs showed similar improving trends. Mean BRTs were faster than preoperative or control values after week 6 (Fig 7). Within the right hip group, only week 8 showed significant findings. No values were statistically significant within the left hip arthroscopy group. In the combined left and right hip arthroscopy group ("all hip BRTs"), week 2 showed a mean BRT near baseline values. Weeks 4 through 6 were, on average, faster than baseline, although only week 8 showed statistically significant findings. There was a trend of improving BRTs in the all hip BRT group (Fig 8).

\section{Discussion}

This systematic review found variations in the recommendations for the return to driving after both hip and knee arthroscopy procedures. Regarding knee arthroscopy, recommendations ranged from 1 week to 6 weeks and were, in part, dependent on the procedure performed and/or laterality of the procedure. For hip arthroscopy, recommendations ranged from 2 weeks to 6 weeks, again depending on laterality for 1 study. Metaanalysis of all knee BRTs showed that at 6 weeks, the 
Table 4. Demographic Characteristics of Participants in Studies Included in Meta-analysis

\begin{tabular}{|c|c|c|c|c|c|c|c|c|c|}
\hline \multirow[b]{2}{*}{ Authors } & \multirow[b]{2}{*}{ Year } & \multirow[b]{2}{*}{ Procedure } & \multirow[b]{2}{*}{ No. of Participants } & \multirow[b]{2}{*}{$\begin{array}{l}\text { No. of Male } \\
\text { Participants }\end{array}$} & \multirow[b]{2}{*}{$\begin{array}{l}\text { No. of Female } \\
\text { Participants }\end{array}$} & \multirow[b]{2}{*}{ Laterality } & \multicolumn{2}{|c|}{ Age, yr } & \multirow{2}{*}{$\begin{array}{c}\text { Measured } \\
\text { Variables Used in } \\
\text { Meta-analysis }\end{array}$} \\
\hline & & & & & & & Surgical & Control & \\
\hline Nguyen et al. ${ }^{21}$ & 2000 & ACL repair & $\begin{array}{l}40 \text { (31 surgical } \\
\text { and } 9 \text { control) }\end{array}$ & 18 & 13 & 16 right and 15 left & $\begin{array}{c}30.2 \pm 7.9 \text { for right } \\
\text { knee and } 30.3 \pm 8.5 \\
\text { for left knee }\end{array}$ & $33.8 \pm 10.8$ & $\begin{array}{l}\text { Right BRT and } \\
\text { left BRT }\end{array}$ \\
\hline Gotlin et al. ${ }^{27}$ & 2000 & ACL repair & $\begin{array}{l}35 \text { (14 surgical } \\
\text { and } 21 \text { control) }\end{array}$ & $\begin{array}{l}12 \text { surgical and } \\
15 \text { control }\end{array}$ & $\begin{array}{l}15 \text { surgical and } \\
15 \text { control }\end{array}$ & Right only & 31.2 & 29.3 & Right BRT \\
\hline Hau et al. ${ }^{20}$ & 2000 & $\begin{array}{l}\text { Partial } \\
\text { meniscectomy, } \\
\text { chondroplasty, } \\
\text { and diagnostic } \\
\text { arthroscopy }\end{array}$ & $\begin{array}{l}55 \text { (30 surgical } \\
\text { and } 25 \text { control) }\end{array}$ & $\begin{array}{l}9 \text { surgical and } 8 \\
\text { control }\end{array}$ & $\begin{array}{l}21 \text { surgical and } \\
17 \text { control }\end{array}$ & Right only & $42.2 \pm 14.2$ & $33.6 \pm 11.0$ & Right BRT \\
\hline Wasserman et al. ${ }^{22}$ & 2017 & ACL repair & $\begin{array}{l}57 \text { (27 surgical } \\
\text { and } 30 \text { control) }\end{array}$ & $\begin{array}{l}12 \text { surgical and } \\
15 \text { control }\end{array}$ & $\begin{array}{l}15 \text { surgical and } \\
15 \text { control }\end{array}$ & Right only & $\begin{array}{c}29.2 \pm 8.2 \text { for } \mathrm{HS} \\
25.0 \pm 4.2 \text { for } \\
\mathrm{BPTB}, \text { and } 44.0 \pm \\
11.4 \text { for } \mathrm{TA}\end{array}$ & $30.4 \pm 3.8$ & Right BRT \\
\hline Valentí et al. ${ }^{24}$ & 2018 & ACL repair & $\begin{array}{l}62 \text { (31 surgical } \\
\text { and } 31 \text { control) }\end{array}$ & $\begin{array}{l}27 \text { surgical and } \\
22 \text { control }\end{array}$ & $\begin{array}{l}7 \text { surgical and } \\
9 \text { control }\end{array}$ & 18 right and 13 left & $32.39 \pm 9.27$ & $28.58 \pm 8.91$ & $\begin{array}{l}\text { Right BRT and } \\
\text { left BRT }\end{array}$ \\
\hline Vera et al. ${ }^{23}$ & 2017 & $\begin{array}{l}\text { Femoroacetabular } \\
\text { impingement } \\
\text { repair and labral } \\
\text { repair }\end{array}$ & $\begin{array}{l}38 \text { (19 surgical } \\
\text { and } 19 \text { control) }\end{array}$ & $\begin{array}{l}9 \text { surgical and } 9 \\
\text { control }\end{array}$ & $\begin{array}{l}10 \text { surgical and } \\
10 \text { control }\end{array}$ & 11 right and 8 left & $\begin{array}{l}37.1 \pm 12.7 \text { for right } \\
\text { knee and } 32.1 \pm 9.2 \\
\quad \text { for left knee }\end{array}$ & $\begin{array}{c}35.5 \pm 11.1 \text { for right } \\
\text { control and } 32.6 \pm \\
8.4 \text { for left control }\end{array}$ & Right BRT \\
\hline Balazs et al. ${ }^{14}$ & 2018 & $\begin{array}{l}\text { Femoroacetabular } \\
\text { impingement } \\
\text { repair }\end{array}$ & $\begin{array}{l}118 \text { (59 surgical } \\
\text { and } 59 \text { control) }\end{array}$ & $\begin{array}{l}32 \text { surgical and } \\
32 \text { control }\end{array}$ & $\begin{array}{l}27 \text { surgical and } \\
27 \text { control }\end{array}$ & 33 right and 26 left & 33.7 & 33.3 & $\begin{array}{l}\text { Right BRT and } \\
\text { left BRT }\end{array}$ \\
\hline Momaya et al. ${ }^{25}$ & 2018 & $\begin{array}{l}\text { Labral repair, } \\
\text { iliopsoas release, } \\
\text { osteoplasty, and } \\
\text { labral } \\
\text { debridement }\end{array}$ & $\begin{array}{l}31 \text { (14 surgical } \\
\text { and } 17 \text { control) }\end{array}$ & $\begin{array}{l}3 \text { surgical and } 4 \\
\text { control }\end{array}$ & $\begin{array}{l}11 \text { surgical and } \\
13 \text { control }\end{array}$ & Right only & $27.39 \pm 9.13$ & $28.35 \pm 5.81$ & $\begin{array}{l}\text { Right BRT and } \\
\text { left BRT }\end{array}$ \\
\hline
\end{tabular}

BPTB, bone-patellar tendon-bone; BRT, brake reaction time; HS, hamstring; TA, tibialis anterior. 
Table 5. Summary of All BRT Data From Studies Included in Meta-analysis

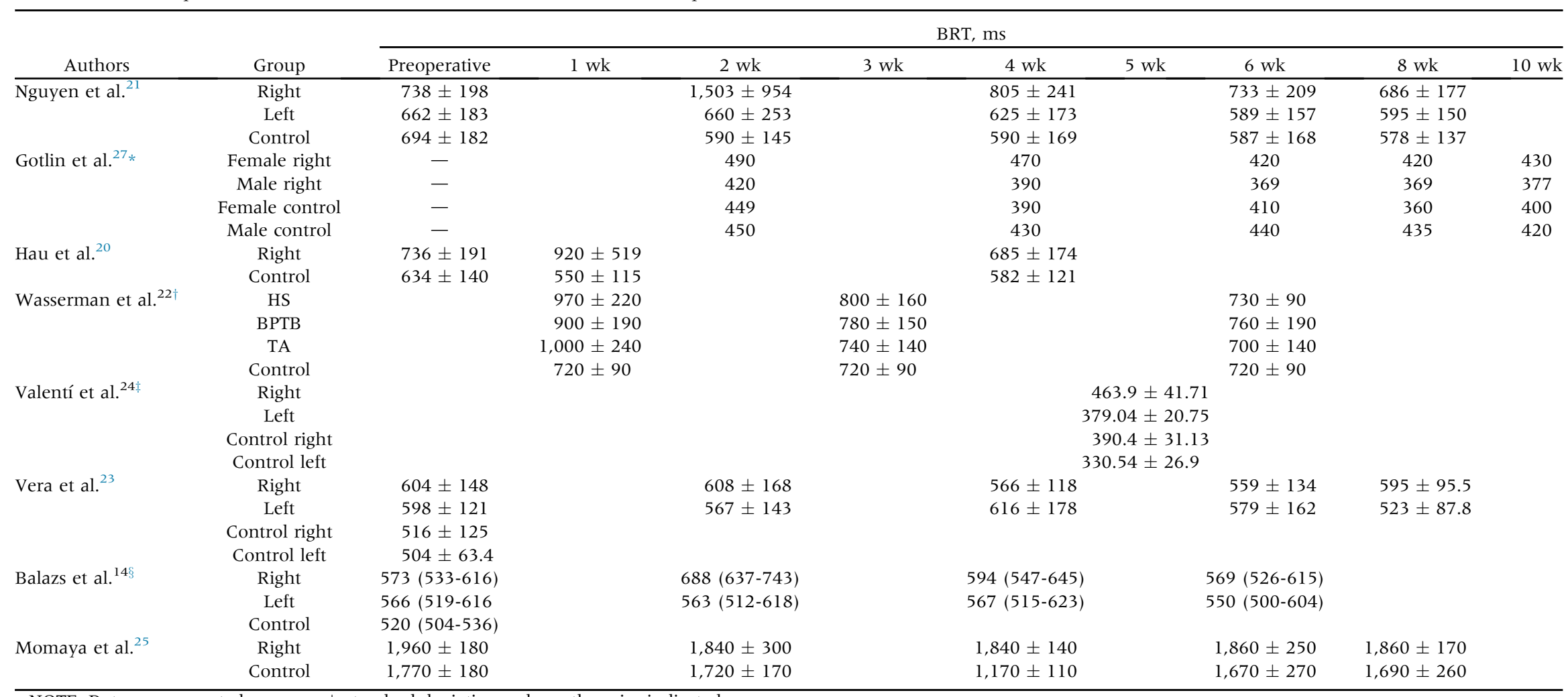

NOTE. Data are presented as mean \pm standard deviation unless otherwise indicated.

BPTB, bone-patellar tendon-bone; BRT, brake reaction time; HS, hamstring; TA, tibialis anterior

*No preoperative values were reported; postoperative BRTs were compared with control values.

${ }^{\dagger}$ All procedures were right sided; groups were categorized by the type of ligament used for reconstruction.

${ }^{\ddagger}$ Postoperative BRTs were collected between weeks 4 and 6.

${ }^{\S}$ Data are reported as mean (confidence interval). 


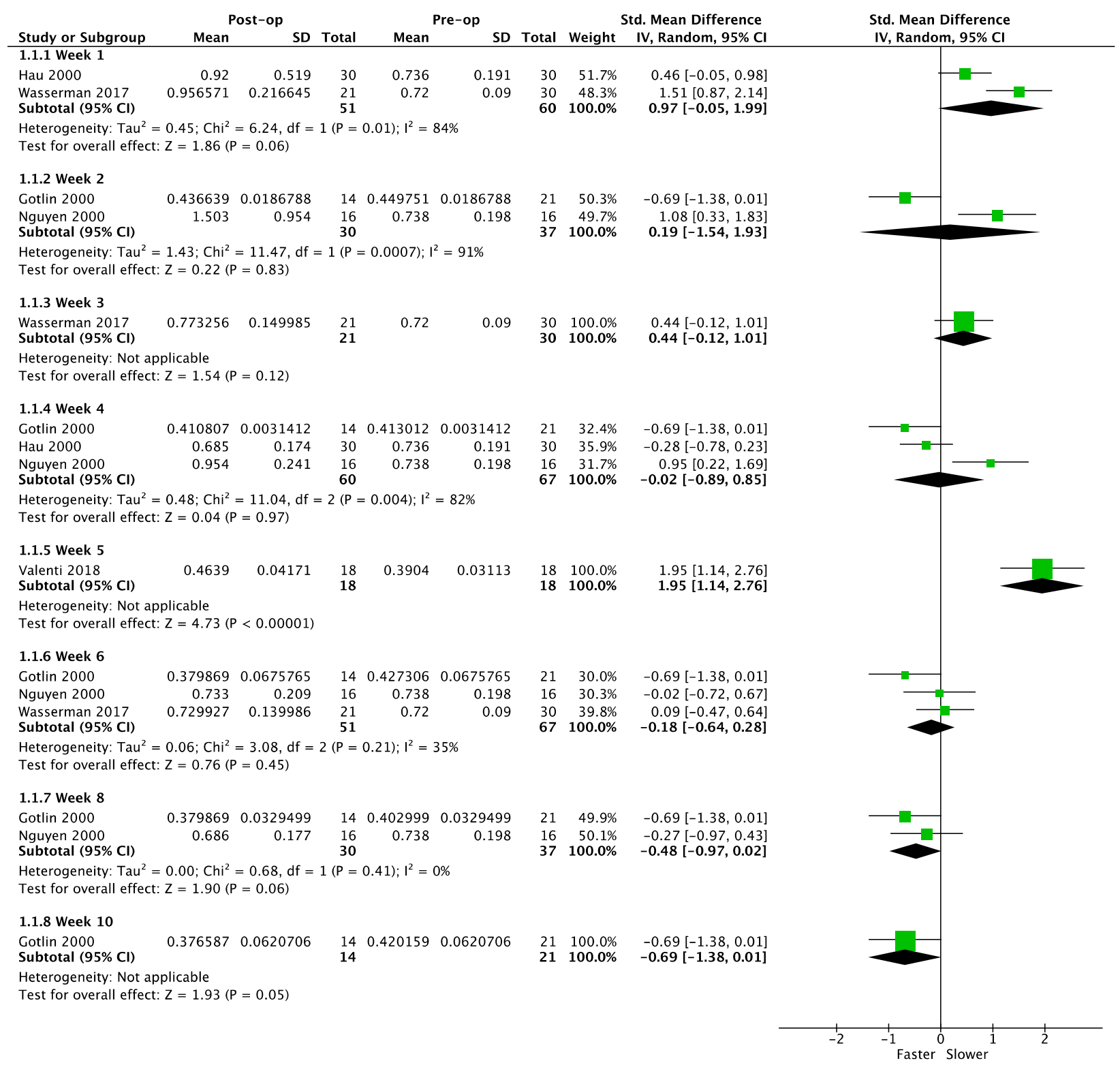

Fig 3. Pooled data and forest plot of right knee arthroscopy brake reaction times. $P<.05$ is considered statistically significant. It should be noted that Valentí et al. ${ }^{24}$ reported brake reaction times between 4 and 6 weeks postoperatively. These data were averaged to "week 5" for the purposes of analysis and plotting data. (CI, confidence interval; IV, inverse-variance method; Postop, postoperative; Pre-op, preoperative; SD, standard deviation; Std, standardized.)

pooled means had met or become faster than the preoperative or control values, and there was a trend of improving BRTs after this point. Side-specific analysis revealed similar findings (patients were able to return more quickly to driving after left knee arthroscopy, but these findings were not statistically significant), with pooled means showing improved BRTs after 6 weeks, as well as a trend of improvement in the right-sided group through week 10. Meta-analysis of all hip BRTs showed that pooled means had met or become faster than preoperative or control times at 4 weeks, with a trend of improving BRTs. It is interesting to note that side-specific analysis revealed that pooled means for right-sided procedures were faster than preoperative or control BRTs at 4 weeks whereas pooled means for left-sided procedures just met baseline values at 4 weeks. These findings are notable because a meta-analysis had not been performed on this subject previously, although the small data pool does suggest the need for further research to expand the pool for future meta-analysis.

When assessing BRT, patients' values in most studies included in the meta-analysis were compared with their preoperative values. This assumes that the patient was driving safely prior to the surgical procedure. However, a patient presenting with the need for arthroscopy presumably has an underlying pathology that requires 


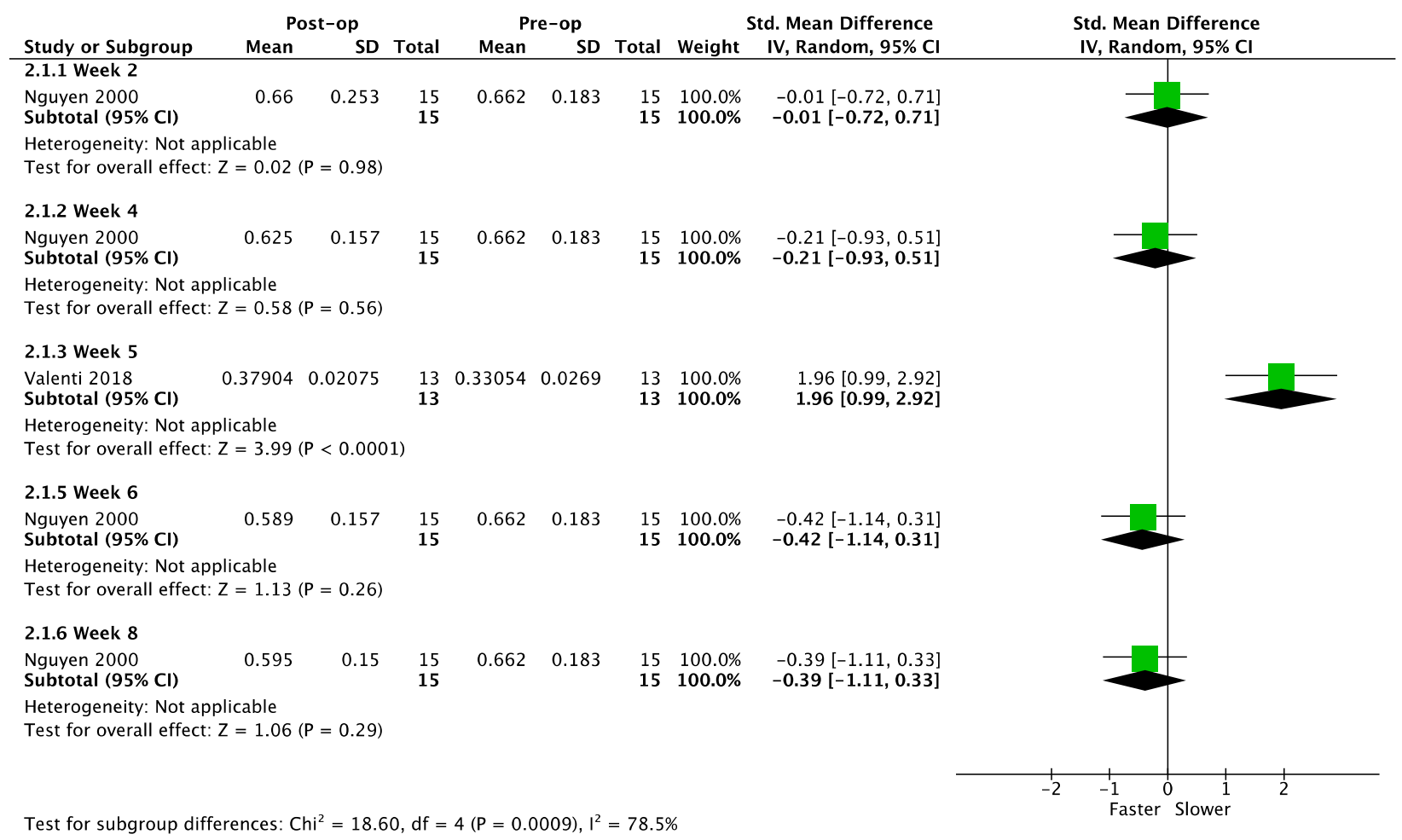

Fig 4. Pooled data and forest plot of left knee arthroscopy brake reaction times. $P<.05$ is considered statistically significant. It should be noted that Valentí et al. ${ }^{24}$ reported brake reaction times between 4 and 6 weeks postoperatively. These data were averaged to "week 5" for the purposes of analysis and plotting data. (CI, confidence interval; IV, inverse-variance method; Post-op, postoperative; Pre-op, preoperative; SD, standard deviation; Std, standardized.)

surgical intervention. The presence of this pathology before surgery could lead to not only slower baseline BRTs but also a decreased ability to drive safely. In this instance, it would be difficult to determine, using BRT alone, whether a patient is able to safely return to driving after surgery. Future studies on BRTs might consider comparing postoperative BRTs with both preoperative values and the corresponding values of a healthy control group.

Both the study by Nguyen et al. ${ }^{21}$ and the study by Hau et al. ${ }^{20}$ had their participants perform sit-to-stand tests in the office and found that there was a significant correlation between performance on sit-to-stand testing and BRTs. This test potentially could be performed during follow-up appointments by surgical patients to assess their readiness to drive. Although BRT is a very important factor to be considered when making recommendations on the resumption of driving, many studies have noted the significance of factoring in other variables, such as cessation of opioid analgesics, strength of the surgical limb, and range of motion. ${ }^{11}$

An interesting consideration related to both preoperative and postoperative driving ability is joint proprioception, particularly related to knee arthroscopy and ACL reconstruction. The ACL itself helps with proprioception of the knee joint, containing many mechanoreceptors that respond to mechanical stimulation and help determine where one's limb is in space. Damage to the ACL before surgery leads to a decrease in proprioceptive ability not only in the surgical knee but also in the contralateral knee, as noted by previous studies. ${ }^{32-34}$ If a patient continues driving, this could lead to an impaired driving ability and slower BRTs preoperatively. However, it is also important to note that reconstruction of the ACL does not lead to an immediate return of proprioceptive ability. Extensive and consistent physical therapy and kinesthetic exercises are needed to allow for the return of proprioceptive ability. ${ }^{32-34}$ Additionally, because autografts (e.g., hamstring or patellar tendon) use tissue from the patients themselves, damage to the donor tissue could lead to a further decrease in proprioceptive ability. ${ }^{35}$

The type of surgical procedure also appeared to be related to the recommended resumption of driving. Studies that exclusively examined ACL reconstruction recommended resumption of driving at 6 weeks or between 4 and 6 weeks. ${ }^{21,22,24,27}$ Studies that examined simple knee arthroscopy, such as those of Hau et al. ${ }^{20}$ and Argintar et al., ${ }^{6}$ gave much earlier recommendations on the resumption of driving (approximately 1 week). This finding highlights that there is a clear difference between knee ligamentous and non-ligamentous surgery and advice should be given to patients accordingly. Likewise, Wasserman et al. $^{22}$ found that, regarding ACL 


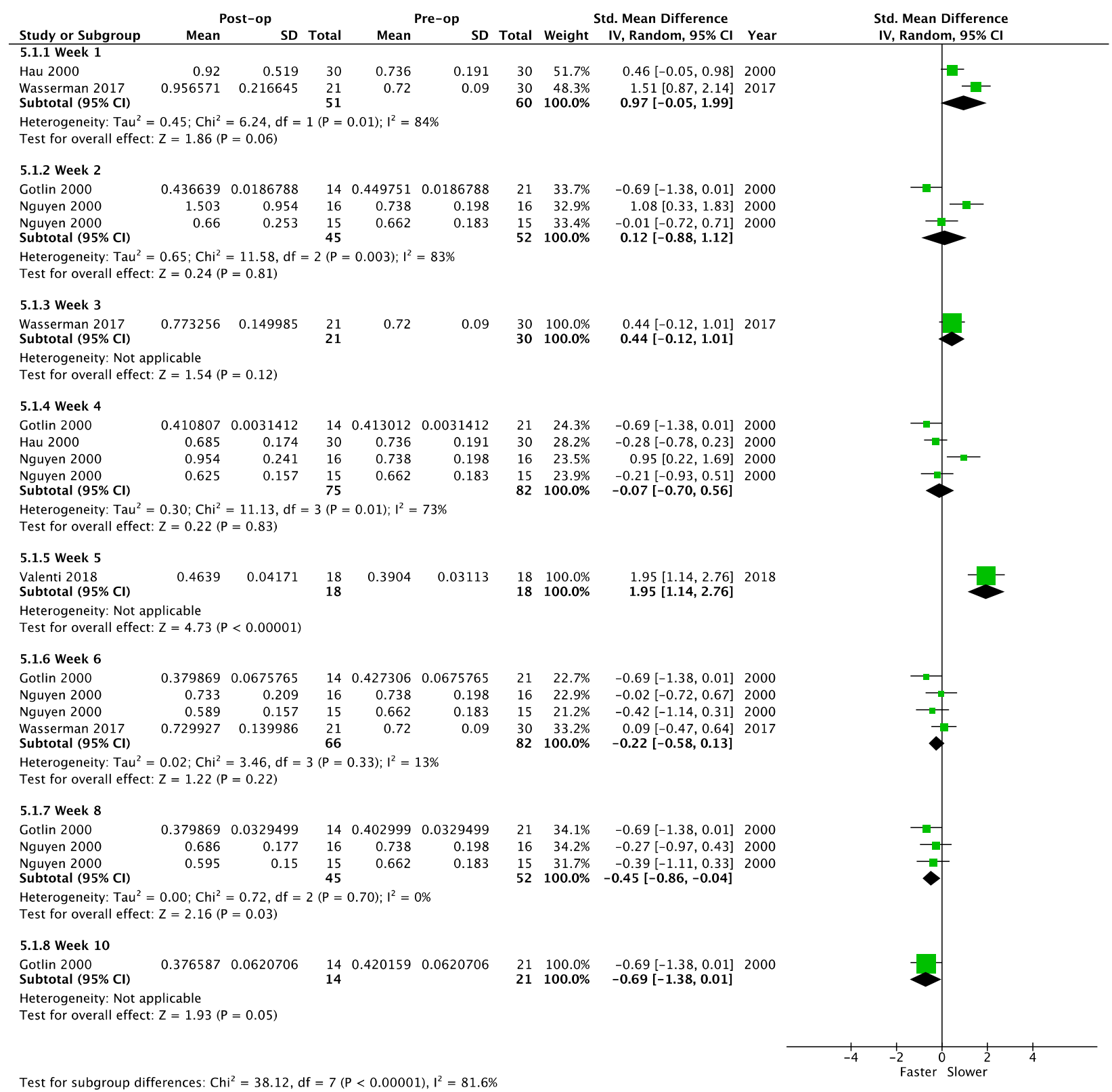

Fig 5. Pooled data and forest plot of all knee arthroscopy brake reaction times. $P<.05$ is considered statistically significant. It should be noted that Valentí et al. ${ }^{24}$ reported brake reaction times between 4 and 6 weeks postoperatively. These data were averaged to "week 5" for the purposes of analysis and plotting data. (CI, confidence interval; IV, inverse-variance method; Post-op, postoperative; Pre-op, preoperative; SD, standard deviation; Std, standardized.)

reconstruction, the specific type of graft had a correlation with when postoperative BRTs met baseline values. On the basis of their results, they recommended resumption of driving at 6 weeks postoperatively after hamstring and bone-patellar tendon-bone autograft procedures and at 3 weeks postoperatively after tibialis anterior allograft procedures. $^{22}$ This provides an additional starting point for future research to explore more deeply.

\section{Limitations}

One notable limitation of this study was the small existing data pool from a limited number of studies.
There is limited existing research regarding the resumption of driving after arthroscopic procedures specifically. Additionally, among the few available studies, there is considerable heterogeneity of data. For instance, the effect of ACL reconstruction on BRTs may not be comparable to the effect of meniscal repair or diagnostic arthroscopy on BRTs. Should the effects on BRTs be separated by type of procedure, given sufficient data, there may be different outcomes and, therefore, different recommendations than those noted in this article. There were also slight methodologic differences between studies, as well as varied outcome measures. 


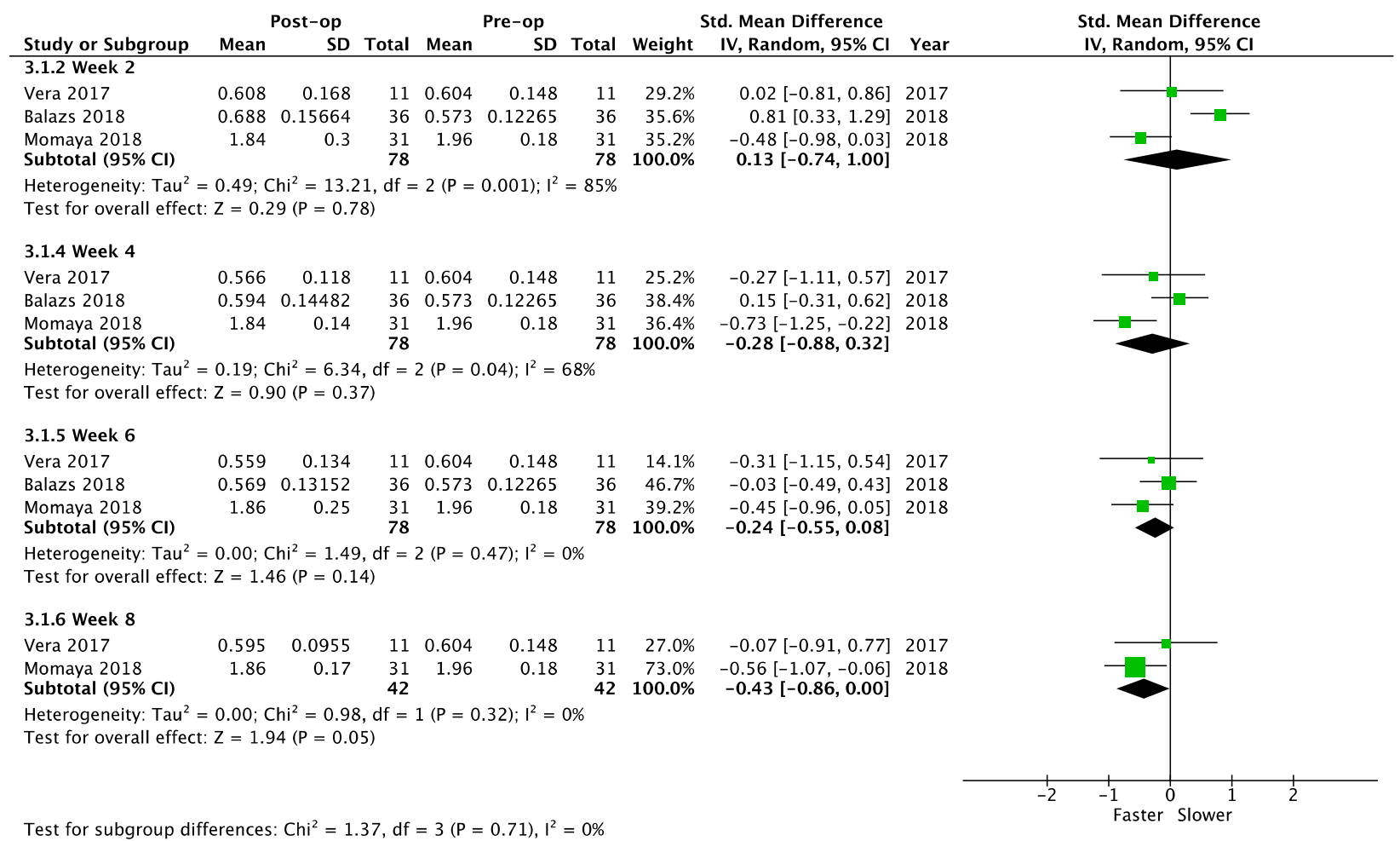

Fig 6. Pooled data and forest plot of right hip arthroscopy brake reaction times. $P<.05$ is considered statistically significant. (CI, confidence interval; IV, inverse-variance method; Post-op, postoperative; Pre-op, preoperative; SD, standard deviation; Std, standardized.)

An example of this is highlighted by the study of Valentí et al., ${ }^{24}$ which collected BRTs between 4 and 6 weeks postoperatively, as opposed to collecting them each week (week 4, week 5, and week 6) as other studies did. This proved to be a challenge when incorporating these data for comparison with data from other studies,

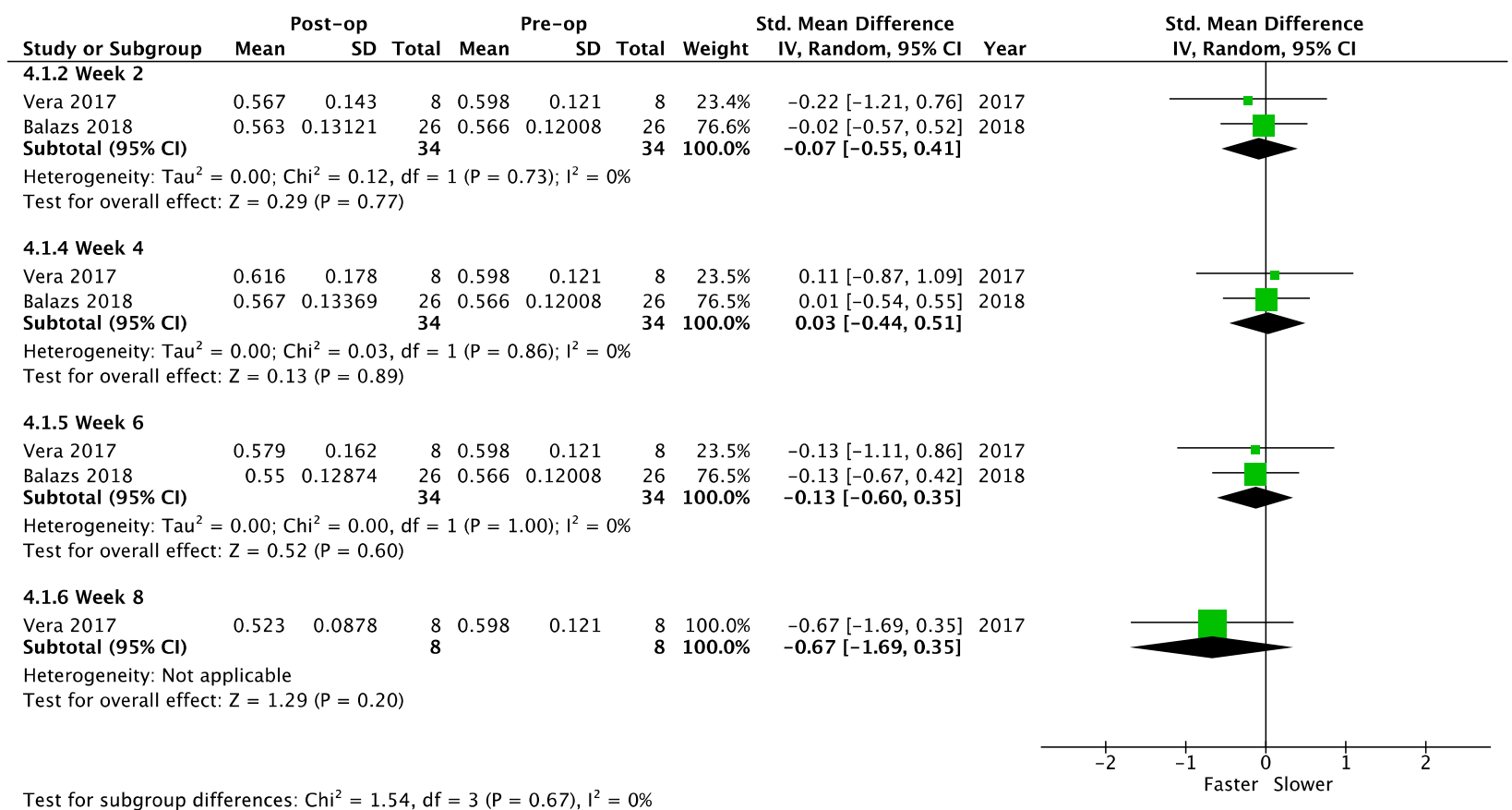

Fig 7. Pooled data and forest plot of left hip arthroscopy brake reaction times. $P<.05$ is considered statistically significant. (CI, confidence interval; IV, inverse-variance method; Post-op, postoperative; Pre-op, preoperative; SD, standard deviation; Std, standardized.) 


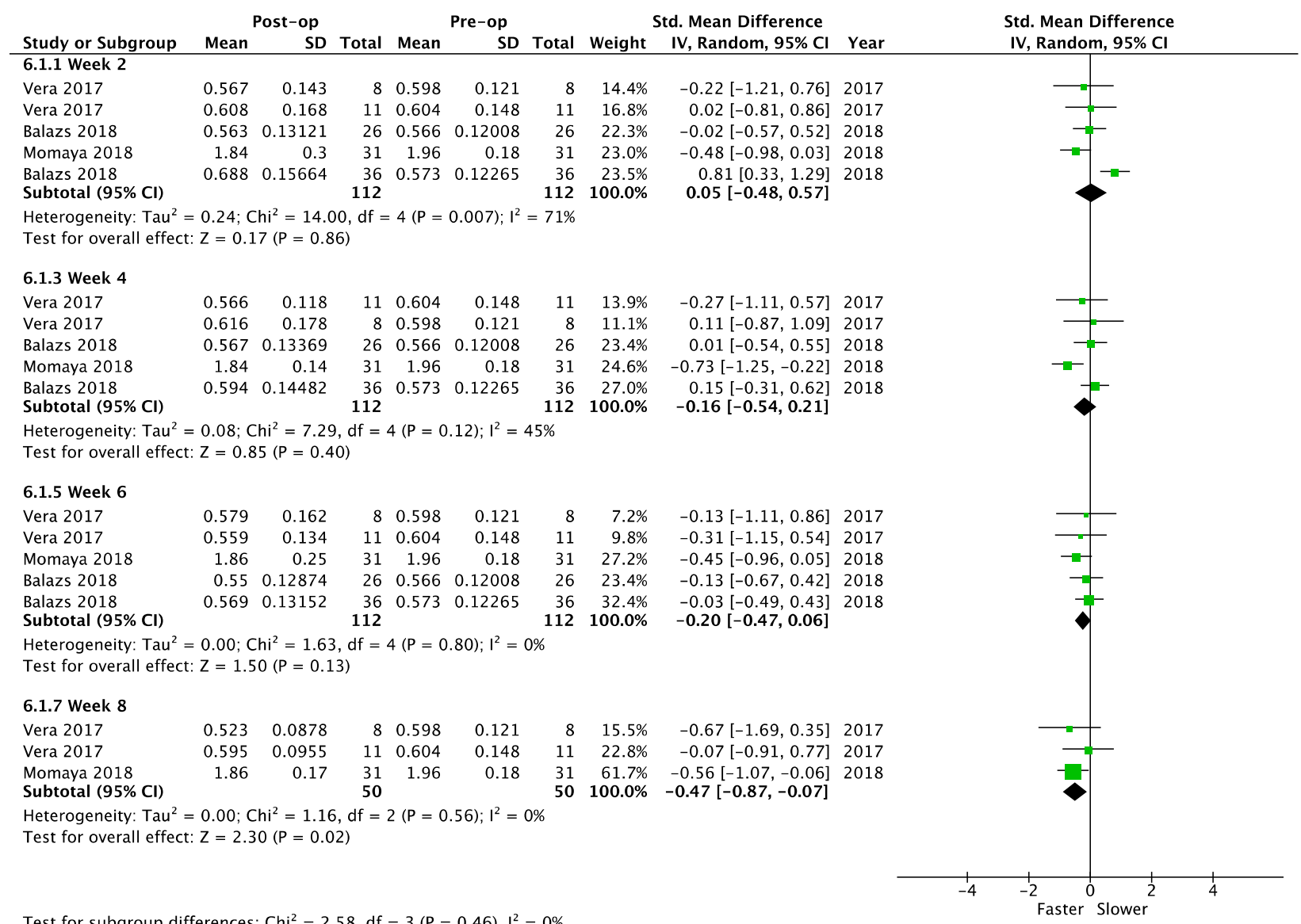

Fig 8. Pooled data and forest plot of all hip arthroscopy brake reaction times. $P<.05$ is considered statistically significant. (CI, confidence interval; IV, inverse-variance method; Post-op, postoperative; Pre-op, preoperative; SD, standard deviation; Std, standardized.)

as the data of Valentí et al. had to be averaged to "week 5 " for the purposes of plotting and statistical analysis. Because of this larger time frame for BRTs, the data could appear skewed: BRTs taken closer to the 4-week mark would appear faster than in actuality, whereas BRTs measured closer to the 6-week mark would appear slower than in actuality.

Our study also suggests the need for further research to expand the available data pool. Further research could more extensively explore BRT differences based on the side of surgery or type of surgical procedure, investigate the effects of non-modifiable factors such as sex and age, or consider additional measures of driving performance.

\section{Conclusions}

BRTs met baseline or control values and continued to improve after 6 weeks after knee arthroscopy and after 4 weeks after hip arthroscopy. On the basis of these results, it would be safe to recommend a return to driving at 6 weeks after knee arthroscopic procedures and 4 weeks after hip arthroscopic procedures.

\section{References}

1. Millenium Research Group. News archive. Hip arthroscopy procedures to soar through 2013. https:// decisionresourcesgroup.com/news/. Published 2009. Accessed November 10, 2020.

2. Kim S, Bosque J, Meehan JP, Jamali A, Marder R. Increase in outpatient knee arthroscopy in the United States: A comparison of National Surveys of Ambulatory Surgery, 1996 and 2006. J Bone Joint Surg Am 2011;93: 994-1000.

3. Treuting R. Minimally invasive orthopedic surgery: Arthroscopy. Ochsner J 2000;2:158-163.

4. Colvin AC, Harrast J, Harner C. Trends in hip arthroscopy. J Bone Joint Surg Am 2012;94:e23.

5. Marecek GS, Schafer MF. Driving after orthopaedic surgery. J Am Acad Orthop Surg 2013;21:696-706.

6. Argintar E, Williams A, Kaplan J, et al. Recommendations for driving after right knee arthroscopy. Orthopedics 2013;36:659-665.

7. Molnar LJ. Factors affecting self-regulatory driving practices among older adults-PubMed. https://pubmed-ncbi-nlmnih-gov.proxy.medlib.uits.iu.edu/24372498/. Accessed November 10, 2020. 
8. MacKenzie JS, Bitzer AM, Familiari F, Papalia R, McFarland EG. Driving after upper or lower extremity orthopaedic surgery. Joints 2019;6:232-240.

9. Ho CP, Furlan AD. Driving following acute lower limb painful events. Pain Manag 2012:2:437-444.

10. Fleury TR, Favrat B, Belaieff W, Hoffmeyer P. Resuming motor vehicle driving following orthopaedic surgery or limb trauma. Swiss Med Wkly 2012;142:w13716.

11. DiSilvestro KJ, Santoro AJ, Tjoumakaris FP, Levicoff EA, Freedman KB. When can I drive after orthopaedic surgery? A systematic review. Clin Orthop Relat Res 2016;474: 2557-2570.

12. Goodwin D, Baecher N, Pitta M, Letzelter J, Marcel J, Argintar E. Driving after orthopedic surgery. Orthopedics 2013;36:469-474.

13. Moher D, Liberati A, Tetzlaff J, Altman DG, PRISMA Group. Preferred reporting items for systematic reviews and meta-analyses: The PRISMA statement. PLoS Med 2009; 6:e1000097.

14. Balazs GC, Donohue MA, Brelin AM, Brooks DI, McCabe MP, Anderson TD. Reaction time and brake pedal depression following arthroscopic hip surgery: A prospective case-control study. Arthroscopy 2018;34:1463-1470.el.

15. Higgins JPT, Thomas J, Chandler J, et al. Cochrane Handbook for Systematic Reviews of Interventions. Ed 2. https://onlinelibrary.wiley.com/doi/book/10.1002/9781 1 19536604. Accessed August 16, 2021.

16. Brice R. CASP checklists. CASP-Critical Appraisal Skills Programme. https://casp-uk.net/casp-tools-checklists/. Accessed November 10, 2020.

17. Slim K, Nini E, Forestier D, Kwiatkowski F, Panis Y, Chipponi J. Methodological index for non-randomized studies (MINORS): Development and validation of a new instrument. ANZ J Surg 2003;73:712-716.

18. Howick J, Chalmers I, Glasziou P, Greenhalgh T, Heneghan C, Liberati A. The Oxford 2011 levels of evidence. Oxford Centre for Evidence-Based Medicine. http://www.cebm.net/index.aspx?o=5653. Accessed November 10, 2020.

19. BMJ Best Practice. What is GRADE?, https://bestpractice. bmj.com/info/us/toolkit/learn-ebm/what-is-grade/. Accessed November 10, 2020.

20. Hau R, Csongvay S, Bartlett J. Driving reaction time after right knee arthroscopy. Knee Surg Sports Traumatol Arthrosc 2000;8:89-92.

21. Nguyen T, Hau R, Bartlett J. Driving reaction time before and after anterior cruciate ligament reconstruction. Knee Surg Sports Traumatol Arthrosc 2000;8:226-230.

22. Wasserman BR, Singh BC, Kaplan DJ, et al. Braking reaction time after right-knee anterior cruciate ligament reconstruction: A comparison of 3 grafts. Arthroscopy 2017;33:173-180.

23. Vera AM, Beauchman N, McCulloch PC, Gerrie BJ, Delgado DA, Harris JD. Brake reaction time after hip arthroscopy for femoroacetabular impingement and labral tear. Arthroscopy 2017;33:971-976.

24. Valentí A, Payo-Ollero J, Pérez-Mozas M, LamoEspinosa JM, Valentí JR. Evaluation of driving skills after anterior cruciate ligament reconstruction with hamstring autograft. Knee 2018;25:790-798.

25. Momaya AM, Stavrinos D, McManus B, Wittig SM, Emblom B, Estes R. Return to driving after hip arthroscopy. Clin J Sport Med 2018;28:299-303.

26. Oliver G, Portabella F, Hernandez JA. A comparative study of the neuromuscular response during a dynamic activity after anterior cruciate ligament reconstruction. Eur J Orthop Surg Traumatol Orthop Traumatol 2019;29: 633-638.

27. Gotlin RS, Sherman AL, Sierra N, Kelly M, Scott WN. Measurement of brake response time after right anterior cruciate ligament reconstruction. Arthroscopy 2000;16: $151-155$.

28. Lewis C, Mauffrey C, Hull P, Brooks S. Knee arthroscopy and driving. Results of a prospective questionnaire survey and review of the literature. Acta Orthop Belg 201 1;77:336-338.

29. Cooper JM. Clinical decision making: Doctor, when can I drive? Am J Orthop (Belle Mead NJ) 2007;36:78-80.

30. Obermeier MC, Sikka RS, Tompkins M, et al. Examination of early functional recovery after ACL reconstruction: Functional milestone achievement and self-reported function. Sports Health 2018;10:345-354.

31. Mei-Dan O, McConkey MO, Knudsen JS, Brick MJ. Bilateral hip arthroscopy under the same anesthetic for patients with symptomatic bilateral femoroacetabular impingement: 1Year outcomes. Arthroscopy 2014;30:47-54.

32. Laboute E, Verhaeghe E, Ucay O, Minden A. Evaluation kinaesthetic proprioceptive deficit after knee anterior cruciate ligament (ACL) reconstruction in athletes. J Exp Orthop 2019;6:6.

33. Reider B, Arcand MA, Diehl LH, et al. Proprioception of the knee before and after anterior cruciate ligament reconstruction. Arthroscopy 2003;19:2-12.

34. Dhillon MS, Bali K, Prabhakar S. Proprioception in anterior cruciate ligament deficient knees and its relevance in anterior cruciate ligament reconstruction. Indian J Orthop 2011;45:294-300.

35. Young SW, Valladares RD, Loi F, Dragoo JL. Mechanoreceptor reinnervation of autografts versus allografts after anterior cruciate ligament reconstruction. Orthop J Sports Med 2016;4:2325967116668782. 


\section{Appendix}

Appendix Table 1. OVID, Embase, and Cochrane Database Search Protocols With Numbers of Results Identified From Search Strategy

1. "Knee arthroscopy" or "Hip arthroscopy" or "arthroscopic knee surgery" or "arthroscopic hip surgery" or "anterior cruciate ligament surgery" or "anterior cruciate ligament reconstruction" or "posterior cruciate ligament repair" or "posterior cruciate ligament reconstruction" or "medial patellofemoral ligament repair" or "medial collateral ligament repair" or "lateral collateral ligament repair" or "knee soft tissue repair" or "hip soft tissue repair" or "soft tissue injury" or "joint loose body" or "fibrocartilage" or "arthroscopy" or "arthroscopy rehabilitation" or "Femoroacetabular Impingement repair" or "Labral tear repair" or "chondroplasty" or "microfracture" or "synovectomy" or "Osteochondral Autograft Transfer System" or "mosaicplasty"

2. "total brake response time" or "reaction time" or "moving time" or "movement time" or "recovery of function"

3. "driving reaction" or "driving skill" or "driving ability" or "driving"

4. 2 or 3

5. 1 and 4

6. Limit 5 to English Language Articles

7. Exp Automobile Driving

8. 5 and 7

171,452

97,430

266,893

2,042

1,945

149

Embase

1. "Knee arthroscopy" or "Hip arthroscopy" or "arthroscopic knee surgery" or "arthroscopic hip surgery" or "anterior cruciate ligament surgery" or "anterior cruciate ligament reconstruction" or "posterior cruciate ligament repair" or "posterior cruciate ligament reconstruction" or "medial patellofemoral ligament repair" or "medial collateral ligament repair" or "lateral collateral ligament repair" or "knee soft tissue repair" or "hip soft tissue repair" or "soft tissue injury" or "joint loose body" or "fibrocartilage" or "arthroscopy" or "arthroscopy rehabilitation" or "Femoroacetabular Impingement repair" or "Labral tear repair" or "chondroplasty" or "microfracture" or "synovectomy" or "Osteochondral Autograft Transfer System" or "mosaicplasty"

2. "total brake response time" or "reaction time" or "moving time" or "movement time" or "recovery of function"

3. "driving reaction" or "driving skill" or "driving ability" or "driving"

4. 2 or 3

5. 1 and 4

6. Limit to English Language Articles

Cochrane

1. ("Knee arthroscopy" or "Hip arthroscopy" or "arthroscopic knee surgery" or "arthroscopic hip surgery" or "anterior cruciate ligament surgery" or "anterior cruciate ligament reconstruction" or "posterior cruciate ligament repair" or "posterior cruciate ligament reconstruction" or "medial patellofemoral ligament repair" or "medial collateral ligament repair" or "lateral collateral ligament repair" or "knee soft tissue repair" or "hip soft tissue repair" or "soft tissue injury" or "joint loose body" or "fibrocartilage" or "arthroscopy" or "arthroscopy rehabilitation" or "Femoroacetabular Impingement repair" or "Labral tear repair" or "chondroplasty" or "microfracture" or "synovectomy" or "Osteochondral Autograft Transfer System" or "mosaicplasty")

2. [AND] "total brake response time" or "reaction time" or "moving time" or "movement time" or "recovery of function" or "driving reaction" or "driving skill" or "driving ability" or "return to driving" or "returned to driving"

3. [AND] Automobile driving

4. 1,2 , and 3
40,342

119,340

118,400

235,595

135

130

5,593

17,352 
Appendix Table 2. GRADE Analysis for Assessment of Quality of Evidence Used in Meta-analysis

\begin{tabular}{|c|c|c|c|c|c|c|c|c|c|c|}
\hline Meta-analysis & $\begin{array}{c}\text { Time Point } \\
\text { of Subanalysis }\end{array}$ & $\begin{array}{c}\text { Standardized } \\
\text { Mean Difference }\end{array}$ & $\begin{array}{c}\text { Confidence } \\
\text { Interval }\end{array}$ & Design $(0-2)$ & $\begin{array}{c}\text { Quality } \\
\text { (0-3, with -1 } \\
\text { for Non-RCT) }\end{array}$ & $\begin{array}{c}\text { Inconsistency } \\
(0-1)\end{array}$ & $\begin{array}{c}\text { Indirectness } \\
(0-1)\end{array}$ & $\begin{array}{c}\text { Imprecision } \\
(0-1)\end{array}$ & $\begin{array}{c}\text { Publication } \\
\text { Bias }(0-1)\end{array}$ & $\begin{array}{c}\text { Overall } \\
\text { Quality (0-9) }\end{array}$ \\
\hline \multicolumn{11}{|l|}{ Knee arthroscopy } \\
\hline \multicolumn{11}{|l|}{ TBRT right } \\
\hline & $\mathrm{l} \mathrm{wk}$ & 0.97 & -0.05 to 1.99 & Low $(-2)$ & Moderate $(-1)$ & Yes $(-1)$ & No & Yes $(-1)$ & Yes $(-1)$ & Very low (3) \\
\hline & $2 \mathrm{wk}$ & 0.19 & -1.54 to 1.93 & Low $(-2)$ & Moderate $(-1)$ & Yes $(-1)$ & No & Yes $(-1)$ & Yes $(-1)$ & Very low (3) \\
\hline & $3 \mathrm{wk}$ & 0.44 & -0.12 to 1.01 & Low $(-2)$ & Moderate $(-1)$ & NA & No & Yes $(-1)$ & Yes $(-1)$ & Low (4) \\
\hline & $4 \mathrm{wk}$ & -0.02 & -0.89 to 0.85 & Low $(-2)$ & Moderate $(-1)$ & Yes $(-1)$ & No & Yes $(-1)$ & Yes $(-1)$ & Very low (3) \\
\hline & $5 \mathrm{wk}$ & 1.95 & 1.14 to 2.76 & Low $(-2)$ & Moderate $(-1)$ & NA & No & No & Yes $(-1)$ & Low (5) \\
\hline & $6 \mathrm{wk}$ & -0.18 & -0.64 to 0.28 & Low $(-2)$ & Moderate $(-1)$ & No & No & Yes $(-1)$ & Yes $(-1)$ & Low (4) \\
\hline & $8 \mathrm{wk}$ & -0.48 & -0.97 to 0.02 & Low $(-2)$ & Moderate $(-1)$ & No & No & Yes $(-1)$ & Yes $(-1)$ & Low (4) \\
\hline & $10 \mathrm{wk}$ & -0.69 & -1.38 to 0.01 & Low $(-2)$ & Moderate $(-1)$ & NA & No & Yes $(-1)$ & Yes $(-1)$ & Low (4) \\
\hline \multicolumn{11}{|l|}{ TBRT left } \\
\hline & $2 \mathrm{wk}$ & -0.01 & -0.72 to 0.71 & Low $(-2)$ & Moderate $(-1)$ & NA & No & Yes $(-1)$ & Yes $(-1)$ & Low (4) \\
\hline & $4 \mathrm{wk}$ & -0.21 & -0.93 to 0.51 & Low $(-2)$ & Moderate $(-1)$ & NA & No & Yes $(-1)$ & Yes $(-1)$ & Low (4) \\
\hline & $5 \mathrm{wk}$ & 1.96 & 0.99 to 2.92 & Low $(-2)$ & Moderate $(-1)$ & NA & No & No & Yes $(-1)$ & Low (5) \\
\hline & $6 \mathrm{wk}$ & -0.42 & -1.14 to 0.31 & Low $(-2)$ & Moderate $(-1)$ & NA & No & Yes $(-1)$ & Yes $(-1)$ & Low (4) \\
\hline & $8 \mathrm{wk}$ & -0.39 & -1.11 to 0.33 & Low $(-2)$ & Moderate $(-1)$ & NA & No & Yes $(-1)$ & Yes $(-1)$ & Low (4) \\
\hline \multicolumn{11}{|l|}{ Hip arthroscopy } \\
\hline & $2 \mathrm{wk}$ & 0.13 & -0.74 to 1.00 & Low $(-2)$ & Moderate $(-1)$ & Yes $(-1)$ & No & Yes $(-1)$ & Yes $(-1)$ & Very low (3) \\
\hline & $4 \mathrm{wk}$ & -0.28 & -0.88 to 0.32 & Low $(-2)$ & Moderate $(-1)$ & Yes $(-1)$ & No & Yes $(-1)$ & Yes $(-1)$ & Very low (3) \\
\hline & $6 \mathrm{wk}$ & -0.24 & -0.55 to 0.08 & Low $(-2)$ & Moderate $(-1)$ & No & No & Yes $(-1)$ & Yes $(-1)$ & Low (4) \\
\hline & $8 w \mathrm{k}$ & -0.43 & -0.86 to 0.00 & Low $(-2)$ & Moderate $(-1)$ & No & No & No & Yes $(-1)$ & Low (5) \\
\hline \multicolumn{11}{|l|}{ TBRT left } \\
\hline & $2 \mathrm{wk}$ & -0.07 & -0.55 to 0.41 & Low $(-2)$ & Moderate $(-1)$ & No & No & Yes $(-1)$ & Yes $(-1)$ & Low (4) \\
\hline & $4 \mathrm{wk}$ & 0.03 & -0.44 to 0.51 & Low $(-2)$ & Moderate $(-1)$ & No & No & Yes $(-1)$ & Yes $(-1)$ & Low (4) \\
\hline & $6 \mathrm{wk}$ & -0.13 & -0.60 to 0.35 & Low $(-2)$ & Moderate $(-1)$ & No & No & Yes $(-1)$ & Yes $(-1)$ & Low (4) \\
\hline & $8 \mathrm{wk}$ & -0.67 & -1.69 to 0.35 & Low $(-2)$ & Moderate $(-1)$ & NA & No & Yes $(-1)$ & Yes $(-1)$ & Low (4) \\
\hline
\end{tabular}

NA, not applicable; RCT, randomised controlled trial; TBRT, total brake reaction time.

*Overall quality was rated as very low for scores of 0 to 3; low, 4 to 5; moderate, 6 to 7; or high, 8 to 9 . 